\title{
In vivo antiviral efficacy and immune dampening effect of zapnometinib emphasize a dual therapeutic potential against COVID-19
}

\section{Yvonne Füll}

Atriva Therapeutics $\mathrm{GmbH}$

\section{Lara Waidele}

Atriva Therapeutics $\mathrm{GmbH}$

\section{Hazem Hamza}

Interfaculty Institute for Cell Biology

\section{Oliver Poetz}

Natural and Medical Science Institute at the University of Tuebingen

\section{Andreas Steinhilber}

Signatope $\mathrm{GmbH}$

Viktoria Anselm

Signatope $\mathrm{GmbH}$

\section{Mark Delany}

Utrecht University

Judith van den Brand

Utrecht University https://orcid.org/0000-0001-8420-5406

\section{Geert van Amerongen}

ViroClinics DDL

\section{Leon de Waal}

Viroclinics Biosciences BV

\section{Stephan Ludwig}

Institute of Virology (IVM), Westfaelische Wilhelms Universitaet, Muenster, Nordrhein-Westfalen, 48149 https://orcid.org/0000-0003-4490-3052

Oliver Planz ( $\nabla$ oliver.planz@uni-tuebingen.de) University of Tuebingen

\section{Article}

Keywords:

Posted Date: February 28th, 2022 
DOI: https://doi.org/10.21203/rs.3.rs-1381607/v1

License: (c) (1) This work is licensed under a Creative Commons Attribution 4.0 International License. Read Full License 
1 In vivo antiviral efficacy and immune dampening effect of zapnometinib emphasize a dual therapeutic potential against COVID-19

3

4 Yvonne Füll ${ }^{1,2}$, Lara Waidele ${ }^{1,2}$, Hazem Hamza ${ }^{1,2,3}$, Oliver Pötz ${ }^{4}$, Andreas Steinhilber ${ }^{4}$, Viktoria 5 Anselm $^{4}$, Mark W. Delaney ${ }^{5}$, Judith M.A. van den Brand ${ }^{5}$, Geert van Amerongen ${ }^{6}$, Leon de $6 \mathrm{Waal}^{6}$, Stephan Ludwig ${ }^{7}$ and Oliver Planz ${ }^{1,2 *}$

1) Interfaculty Institute for Cell Biology, Department of Immunology, Eberhard Karls University Tuebingen, Germany

2) Atriva Therapeutics GmbH, Tuebingen, Germany

3) Virology Laboratory, Environmental Research Division, National Research Centre, Cairo, $12 \quad$ Egypt

4) SIGNATOPE GmbH, Reutlingen, Germany

5) Division of Pathology, Faculty of Veterinary Medicine Utrecht, University Utrecht, the Netherlands

6) Viroclinics Xplore, Schaijk, the Netherlands

7) Institute of Virology (IVM), Centre for Molecular Biology of Inflammation, University of Muenster, Muenster, Nordrhein-Westfalen, Germany

*Corresponding author: $\quad$ Prof. Dr. Oliver Planz 
Coronaviruses, in particular the current pandemic strains of SARS-CoV-2, represent an everevolving threat to human life. Currently available antiviral drugs are not suitable for severely infected patients in later hyperinflammatory stages of the disease. New therapeutics that inhibit

31 virus propagation and target inflammation would be ideal. We demonstrate that the host targeted MEK inhibitor zapnometinib displays such a dual effect. The drug efficiently reduced virus titers of different SARS-CoV-2 variants and other highly pathogenic human coronaviruses such as SARS-CoV-1 and MERS-CoV in vitro. In a Syrian hamster infection model, zapnometinib reduced SARS-CoV-2 viral load and alleviated lung pathology. Drug safety biomarkers, body weight change and clinical observations, indicated that zapnometinib was well tolerated and showed no toxicity. Moreover, the immune dampening effect could be confirmed in an acute lung injury mouse model and in vitro experiments in primary human blood cells that demonstrated the reduction of disease related cytokines and chemokines. Thus, in contrast to direct-acting antivirals, zapnometinib displays a dual therapeutic effect

41 highlighting its potential in the treatment of severe COVID-19 and other acute viral infections 42 leading to hyperinflammation. 


\section{INTRODUCTION}

In late 2019, severe acute respiratory syndrome coronavirus 2 (SARS-CoV-2), which causes coronavirus disease 2019 (COVID-19), emerged in humans. The WHO declared COVID-19 as a pandemic due to the rapid person-to-person transmission of SARS-CoV-2 and substantial morbidity and mortality worldwide ${ }^{1-3}$. Severity of COVID-19 varies widely from asymptomatic infection to severe disease, with the latter being associated with lower respiratory tract symptoms and pathological hyperinflammation ${ }^{4-6}$ that may lead to multiorgan dysfunction, including acute respiratory distress syndrome (ARDS), neurological symptoms, cardiovascular events, and coagulopathies ${ }^{7-9}$. While the viral load is high at onset and in the early stages of COVID-19, the hyperinflammatory response plays a more important role in determining disease severity in later stages ${ }^{10}$. Hyperinflammation is evidenced by highly elevated levels of certain proinflammatory cytokines and chemokines that are observed in most COVID-19 patients, especially in patients with severe disease who require intensive care ${ }^{11}$.

Currently available treatments can be grouped in therapeutic antibodies, repurposed directacting antivirals, such as nirmatrelvir, remdesivir, or molnupiravir, and immunomodulating drugs, such as dexamethasone or anti-IL-6 antibodies ${ }^{12-16}$. They all have their limitations. Therapeutic antibodies became inefficient because of the emergence of new SARS-CoV-2 variants, highlighting the problem of resistance. Direct-acting antivirals must be applied very early in the onset of infection and were not very efficient in treating severely ill patients. Immunomodulators such as dexamethasone showed quite good efficacy in late-stage infections, however, should not be given too early to allow the initial onset of an antiviral immune response. Thus, a drug that is effective throughout the full course of the disease is still missing. Targeting cellular factors represents a new strategy to overcome this gap.

The cellular Raf/MEK/ERK signaling pathway is mainly involved in cell proliferation and the regulation of cytokine and chemokine expression ${ }^{17}$. Moreover, inhibition of this pathway leads to modulation of the adaptive immune response toward an effector immune response ${ }^{18-20}$. Thus, 
inhibition of this pathway may interfere with overshooting immune responses. In addition, we previously demonstrated that targeting the cellular Raf/MEK/ERK signaling pathway leads to viral load reduction and protects mice from a lethal viral challenge during infection with another live threatening respiratory virus, the influenza virus ${ }^{21-23}$. This prompted us to analyze whether inhibition of the pathway would also lead to inhibition of SARS-CoV-2 replication. Indeed we could show that zapnometinib (ATR-002, PD 0184264), an oral MEK inhibitor, not only demonstrated antiviral activity against influenza virus ${ }^{22,23}$ but also against SARS-CoV-2 in vitro ${ }^{24}$. Interestingly, the inhibitor also led to the reduction of expression of some virus-induced cytokines in infected cells ${ }^{24}$, although the relevance of this observation has still to be examined in vivo. Additionally, zapnometinib showed no toxicity and was well tolerated in humans in a phase 1 study in healthy volunteers (NCT04385420). The drug is currently being investigated in a phase 2 clinical trial of hospitalized COVID-19 patients (NCT04776044).

Here, we aimed to assess the therapeutic potential of zapnometinib against various coronaviruses, demonstrating its dual effect in vivo with different approaches. The pharmacokinetics, antiviral efficacy, immunomodulatory effect, and biomarker-indicated safety of zapnometinib could be demonstrated in a Syrian hamster SARS-CoV-2 infection model. When administered orally as a single dose starting 4 or $24 \mathrm{~h}$ after infection with SARSCoV-2, zapnometinib significantly decreased viral load and alleviated lung pathology without exerting toxic effects. This was reflected by an apparent lack of treatment-related side effects, body weight changes or changes in the levels of drug safety biomarkers. Furthermore, we revealed that zapnometinib could significantly alleviate hyperinflammation induced by cytokines and chemokines associated with severe COVID-19 in an acute lung injury mouse model and in primary human blood cells. These findings support the potential of zapnometinib as a promising broad-spectrum antiviral therapeutic with a dual effect, particularly in severe viral infections leading to hyperinflammation like COVID-19. 


\section{MATERIALS AND METHODS}

97

\section{Ethics}

The hamster experiments were carried out in the Central Animal Facilities of Viroclinics Xplore in Schaijk, the Netherlands, in accordance with the standards of the Dutch law for animal experimentation (2010/63/EU). Ethical approval for the present study was obtained from the institutional Animal Welfare Body under the number AVD277002015283-WP24.

All mouse experiments were conducted following review and approval by the Regional Council Tuebingen (35/9185.81-2/ IM 1/17) and in compliance with the institutional Principles of Animal Welfare and Animal Research.

\section{Animals}

Seven- to nine-week-old male Syrian hamsters (Janvier, France) with a body weight of 102$134 \mathrm{~g}$ at the time of drug administration were used for the pharmacokinetic study. The same animals were used for the efficacy study at an age of 11-13 weeks and a body weight of 113$148 \mathrm{~g}$. The animals were housed in elongated type 2 IVC group cages with two animals per cage under BSL-II conditions during acclimatization and in elongated type 2 group cages under BSL-III conditions (isolators) during challenge using sawdust as bedding. They were checked daily for overt signs of disease, were fed standard food, and drinking water was provided ad libitum. All biotechnical procedures were performed under 3\% isoflurane anesthesia.

Six- to eight-week-old female C57BL76J mice weighing 20-27 g from the Mouse Facility of the University of Tuebingen (Interfaculty Institute for Cell Biology) were used to establish an inflammatory acute lung injury (ALI) model. The animals were maintained in elongated type 2 IVC cages housing four mice each under BSL-II conditions, fed standard food, and drinking water was provided ad libitum. 
122 SARS-CoV-2 (BetaCoV/Munich/BavPat1/2020, containing the D614G substitution in the S1

123 fragment, kindly provided by Dr. C. Drosten, Berlin, Germany) was passaged once in Vero124 TMPRSS2 cells and three times in Vero E6 cells and titered in a TCID 50 assay as described in 125 the section "Determination of replication-competent virus (TCID 50 assay)". Vero E6 (Cat\# 126 CRL-1586) and Vero cells (Cat\# CCL-81) used for in vitro assays were obtained from ATCC 127 (Manassas, Virginia, USA). The virus strains used for in vitro studies at a MOI of 0.001 were 128 the following: SARS-CoV-1: SARS-CoV-HKU-39849; MERS-CoV: MERS-CoV-EMC/2012; 129 SARS-CoV-2 BavPat1: Human 2019-nCoV Isolate, EVAg Ref-SKU:026V-03883; SARS130 CoV-2 (Alpha / B.1.1.7): Isolate USA/CA_CDC_5574/2020, BEI Resources NR-54011; 131 SARS-CoV-2 (Beta / B.1.351): Isolate hCoV-19/South Africa/KRISP-K005325/2020, BEI 132 Resources NR-54009.

133 Human peripheral blood mononuclear cells (PBMCs) were isolated from healthy volunteers 134 registered with the Biobank of the Department of Immunology at the University of Tuebingen. 135 Ethical approval was obtained upon review by the Ethics Board of the Medical Faculty of the 136 Eberhard Karls University Tuebingen and the University Hospital Tuebingen (887/2020BO2).

137 Briefly, $50 \mathrm{ml}$ whole blood (roughly $5 \times 10^{7} \mathrm{PBMCs}$ ) of two donors were taken in $50 \mathrm{ml}$ syringes 138 (BD Biosciences, Franklin Lakes, New Jersey, USA) supplied with heparin-natrium (100.000 139 I.E./ml; Braun, Melsungen, Germany). The whole blood was mixed with PBS (Gibco, Carlsbad, 140 California, USA) in a 1:1 ratio in $50 \mathrm{ml}$ tubes (Greiner Bio-One, Frickenhausen, Germany), and 141 PBMCs were subsequently isolated from the whole blood by density gradient centrifugation 142 using Histopaque ${ }^{\circledR}-1077$ (Sigma-Aldrich, St. Louis, Missouri, USA) as density gradient 143 medium. $5 \mathrm{ml}$ plasma per donor were preserved for cell culture medium preparation. PBMCs 144 were then purified by washing twice with PBS. 
147 The in vitro antiviral activity assay was performed at Viroclinics Biosciences BV (Rotterdam,

148 the Netherlands) according to the company's standard operating procedures (SOPs). Briefly,

149 Vero E6 cells (for SARS-CoV-2) or Vero cells (for Middle East respiratory syndrome (MERS)-

$150 \mathrm{CoV}$ and SARS-CoV-1) were seeded at a density of $1.5 \times 10^{4}$ cells in 96 -well plates the day

151 before infection and inoculated with $0.001 \mathrm{MOI}$ of appropriate virus in the absence (virus

152 control wells) and presence of $100,75,50,25$ or $0 \mu \mathrm{M}$ zapnometinib. The 96 -well plates were

153 incubated for 24 and $48 \mathrm{~h}$ at $37^{\circ} \mathrm{C}$. The supernatants were harvested after the incubation, and

154 the viral titer was determined in 4 replicates by TrueBlue Immunostaining and spot counting

155 using a CTL Immunospot Image Analyzer. The viral titer measured in the presence of each

156 concentration of zapnometinib was used to determine the $50 \%$ effective concentration $\left(\mathrm{EC}_{50}\right)$

157 using a previously described method ${ }^{25}$.

\section{Drug administration}

Zapnometinib was synthesized by Chemcon $\mathrm{GmbH}$ (Freiburg, Germany). Before administration to Syrian hamsters, it was freshly dissolved in dimethyl sulfoxide (DMSO) (Carl

Roth GmbH, Karlsruhe, Germany) and then further dissolved in 15\% Kolliphor EL (Sigma-

163 Aldrich, Germany) and 80\% phosphate-buffered saline (PBS) (Gibco, Life Technology Europe

B.V., Bleiswijk, the Netherlands). Zapnometinib was administered orally at the indicated doses in a volume of $500 \mu \mathrm{L} / 150 \mathrm{~g}$ body weight using a gavage needle.

\section{Pharmacokinetics}

168 Drug administration and blood sampling in Syrian hamsters were performed by Viroclinics

169 Xplore (Schaijk, the Netherlands) and are described in the sections "Drug administration" and

170 “Sampling after inoculation”. Specifically, at different time points $(0,0.5,1,2,4,8,12$, and 24

$171 \mathrm{~h})$ after the administration of a single dose of zapnometinib (15, 30, or $60 \mathrm{mg} / \mathrm{kg}$ per oral (p.o.)),

$172 \sim 200 \mu \mathrm{L}$ blood was collected retro-orbitally from three animals per time point per dose and 
173 alternating animals between time points (except at $24 \mathrm{~h}, \mathrm{n}=6$ per group) under isoflurane

174 anesthesia using a heparinized capillary tube containing a clot activator (Microvette 500 Z-Gel,

175 Sarstedt). Serum was collected by spinning the tubes at $4000 \mathrm{xg}$ for 10 minutes, aliquoted and

176 stored at $<-70{ }^{\circ} \mathrm{C}$ until pharmacokinetic analysis of serum. Clinical observations were

177 conducted daily and included ruffled fur, hunched back posture, accelerated breathing and

178 lethargy, and the body weight was monitored before and $24 \mathrm{~h}$ post administration.

179 Bioanalysis and pharmacokinetic evaluation were then performed at Prolytic GmbH (Frankfurt,

180 Germany). High-affinity liquid chromatography tandem mass spectrometry (HPLC-MS/MS)

181 was used to determine the zapnometinib concentration in hamster serum according to the

182 company's SOPs. During the analytical phase, two batches were evaluated. The acceptance

183 criteria for the calibration standards and quality control (QC) samples were fulfilled for both

184 batches according to the company's SOPs. The calibration curve was in the range of 10-10000

$185 \mathrm{ng} / \mathrm{mL}$ for zapnometinib and showed acceptable linearity over the calibration range. In total, 81

186 study test samples were analyzed. The validity of the method during the analysis of the test

187 samples was ensured by assaying QC samples with known concentrations of zapnometinib;

188 zapnometinib 13C6 was used as the internal standard. The lower limit of quantification (LLOQ)

189 of the method was determined to be $10 \mathrm{ng} / \mathrm{mL}$ for zapnometinib in undiluted serum. ANALYST

190 software was used to calculate the concentrations of the test and QC samples based on the

191 corresponding calibration curves. The obtained mean serum concentrations were used for

192 noncompartmental pharmacokinetic evaluation with Phoenix ${ }^{\circledR}$ WinNonLin software

193 (Pharsight Corporation, Mountain View, CA, USA; version 8.1).

195 Antiviral efficacy study in the Syrian hamster infection model

196 On day 0 , all animals were infected intranasally with SARS-CoV-2 (1x $10^{3}$ median tissue culture

197 infectious dose (TCID 50 ) BetaCoV/Munich/BavPat1/2020 (grown and titered at Viroclinics) in

198 a total volume of $0.1 \mathrm{~mL}$ diluted in PBS (Gibco, Paisley, UK) and divided between the nostrils. 
Zapnometinib (100 mg/kg) was administered p.o. at 4 or $24 \mathrm{~h}$ post-infection (p.i.). Thereafter,

200 the animals in both groups were treated with $75 \mathrm{mg} / \mathrm{kg}$ zapnometinib once daily. The animals 201 in the control group received vehicle $4 \mathrm{~h}$ p.i. and once daily thereafter. All animals 202 were euthanized by exsanguination under 3\% isoflurane anesthesia on day 4 post-infection 203 (dpi).

\section{Sampling after inoculation}

Respiratory tract samples were collected daily during the study from Syrian hamsters infected and treated as described in the in the section "Antiviral efficacy study in the Syrian hamster 208 infection model”. Briefly, throat swabs were collected in virus transport medium (Eagles 209 minimal essential medium containing HEPES buffer, Na bicarbonate solution, L-Glutamine, 210 Penicillin, Streptomycin, BSA fraction V and Amphotericin B), and the samples were aliquoted 211 and stored at $<-70{ }^{\circ} \mathrm{C}$. Serum was also collected as described for pharmacokinetics at 0 dpi and 2124 dpi and frozen at $<-70{ }^{\circ} \mathrm{C}$ until further analysis.

213 Additionally, upon necropsy, lung, nasal turbinates and trachea samples were collected and 214 stored in $10 \%$ formalin for histopathology or frozen at $<-70{ }^{\circ} \mathrm{C}$ for virological analysis. For 215 virological analysis, the tissue samples were weighed, homogenized in infection medium and centrifuged at $3000 \mathrm{xg}$ for 10 minutes at room temperature before titration.

\section{Determination of viral load}

219 Detection of replication-competent virus (TCID50 assay): Quadruplicate 10-fold serial 220 dilutions of throat swap and tissue homogenate samples were used to determine the viral titers 221 in confluent layers of Vero E6 cells. Briefly, serial dilutions of samples were prepared and 222 incubated on Vero E6 monolayers for $1 \mathrm{~h}$ at $37^{\circ} \mathrm{C}$. Then, the Vero E6 monolayers were washed 223 once with infection medium (DMEM containing L-Glutamine, Penicillin, Streptomycin, 224 Amphotericin B and Fetal Bovine serum) and incubated for 4-6 days at $37^{\circ} \mathrm{C}$, after which the 
plates were scored using WST8 (colorimetric assessment). The optical density (OD) of the wells was read at $450 \mathrm{~nm}\left(\mathrm{OD}_{450}\right)$ using a microplate reader and compared to the OD of the positive control wells (non-treated, infected cells), which showed a cytopathic effect (CPE). The viral titer $\left(\mathrm{TCID}_{50}\right.$ ) per $\mathrm{mL}$ or $\mathrm{g}$ was calculated using the Spearman-Karber method.

Detection of viral RNA: The viral titer in throat swab and homogenized tissue samples was

230 determined using an RT-qPCR method. Briefly, RNA was isolated with a Roche MagNA Pure 23196 instrument using a MagNA Pure 96 DNA and Viral NA Small Volume Kit, and a TaqMan232 based RT-qPCR assay was performed with specific primers (Supplementary Table 1), as 233 described by Corman et al. ${ }^{26}$. The number of viral copies (CP per $\mathrm{mL}$ or $\mathrm{g}$ ) per sample was 234 calculated by using the resulting Ct value of the sample and the slope, intercept, and upper and 235 lower limits of detection of a $\log _{10}$ dilution series of a viral stock for which the number of copies 236 per dilution was known.

\section{Gross pathology}

239 At the time of necropsy, gross pathology was performed for each animal. All lung lobes were 240 inspected, and the percentage of affected lung tissue was estimated from the dorsal view. In 241 addition, any other abnormalities observed in other organs during full-body gross pathology were recorded. Then, the left lung lobes, trachea and nasal turbinates were preserved in $10 \%$ neutral buffered formalin for histopathology, and the right lung lobes and nasal turbinates were subsequently homogenized for TaqMan-based RT-qPCR and virus titration.

\section{Histopathology}

247 Histopathological analysis of the left lung, trachea and left nasal turbinate was performed for 248 all animals. After fixation with $10 \%$ formalin for at least 2 weeks, tissue sections were 249 embedded in paraffin and stained with hematoxylin and eosin (H\&E) for histological 250 examination. SARS-CoV-2-associated lesions in the lungs were semi-quantitatively assessed 
as follows: presence of alveolar edema, alveolar hemorrhage, and type II pneumocyte

252 hyperplasia: $0=$ no, and $1=$ yes.

\section{Candidate biomarker analysis}

255 Serum samples from hamsters representing pre- $(0 \mathrm{dpi})$ and post-infection (4 dpi) and treatment 256 timepoints were analyzed to assess the levels of different candidate safety biomarkers. Serum 257 proteins were enzymatically fragmented by proteolysis, and protein-specific peptides were 258 targeted for quantification of the corresponding protein biomarker levels by immunoaffinity 259 liquid chromatography tandem mass spectrometry (IA-LC-MS/MS) performed as described 260 previously ${ }^{27,28}$. For details see supplementary.

\section{Inflammatory ALI mouse model}

263 Mice were anesthetized by intraperitoneal (i.p.) injection of ketamine $(10 \mathrm{mg} / \mathrm{kg})$ solution. The 264 mice in both the treatment and control groups $(\mathrm{n}=4$ each) were treated with $5 \mathrm{mg} / \mathrm{kg}$ LPS 265 (O55:B5, Sigma-Aldrich) in PBS (100 $\mu \mathrm{L}$ per mouse) via i.p. injection. At $1 \mathrm{~h}$ post-stimulation, 266 the mice in the treatment group were treated with $25 \mathrm{mg} / \mathrm{kg}$ zapnometinib via i.p injection, 267 while the mice in the control group were treated with vehicle (1\% DMSO in PBS). The mice were euthanized at $6 \mathrm{~h}$ post-zapnometinib treatment, and the lungs were preserved in RNAlater 269 (Qiagen, Hilden, Germany).

270 Lungs were homogenized by a bead beating system (FastPrep ${ }^{\mathrm{TM}}$, MP Biomedicals) and the 271 RNA was isolated using an RNeasy Plus Universal Midi Kit (Qiagen) according to the 272 manufacturer's instructions. RNA quality (A260/A280 ratio $\geq 1.8$ and A260/A230 in the range of 2.0-2.2) was determined using a NanoDrop spectrophotometer (Thermo Fisher Scientific),

274 and the isolated RNA was reverse transcribed using an RT $^{2}$ First Strand Kit (Qiagen). 275 Afterward, the generated cDNA was subjected to a real-time RT ${ }^{2}$ Profiler PCR Array using RT ${ }^{2}$ 276 SYBR ${ }^{\circledR}$ Green qPCR Mastermix. For optimal performance, the - RT $^{2}$ Profiler ${ }^{\mathrm{TM}}$ Mouse 
277 Cytokines \& Chemokines arrays (PAMM-150ZR, Qiagen) were customized to include 84 278 genes and 5 housekeeping genes for data normalization (see Supplementary Table 3). For QC, 279 a mouse genomic DNA contamination test, 3 reverse transcription efficiency tests, and 3 PCR 280 array reproducibility tests were included. The data were analyzed using the GeneGlobe portal 281 (Qiagen) according to the $\Delta \Delta \mathrm{Ct}$ method. The fold change/regulation was calculated, and the 282 threshold value was 2.

\section{Analysis of cytokine and chemokine levels in human PBMCs}

$2 \times 10^{6}$ human PBMCs from two donors each were suspended in $1 \mathrm{~mL}$ RPMI 1640 medium (Gibco) containing 5\% autologous plasma, 1\% penicillin/streptomycin (Sigma-Aldrich), and

1\% L-glutamine (Sigma-Aldrich) and were treated with $1 \mu \mathrm{g} / \mathrm{mL}$ LPS from E. coli O55:B5 (prepared in PBS) and $10 \mu \mathrm{g} / \mathrm{mL}$ zapnometinib (prepared in DMSO and diluted in cell culture medium to a final DMSO concentration of $0.1 \%$ ) for $6 \mathrm{~h}$ at $37{ }^{\circ} \mathrm{C}$ and $5 \% \mathrm{CO}_{2}$. The supernatants (cell culture medium) were collected and stored at $-20{ }^{\circ} \mathrm{C}$ until analysis by ELISA. Ready-touse ELISA kits (Invitrogen, Waltham, Massachusetts, USA) were used according to the manufacturer's instructions to determine the levels of the following cytokines: IL-1 $\beta$, IL-6, IL8, IP-10, MCP-1, MIP-1 $\alpha$, and TNF- $\alpha$. Briefly, the samples and standards were added to ELISA plates precoated with specific antibodies against the cytokines of interest. Biotinylated detection antibodies binding to the cytokines of interest were added to the wells followed by a horseradish peroxidase (HRP)-streptavidin conjugate binding to the biotinylated detection antibodies. Unbound cytokines, detection antibodies, and HRP-streptavidin conjugate were 298 removed by washing. Tetramethylbenzidine (TMB) substrate was added and the color change 299 in the wells was used as readout for the ELISA. The optical density (OD) at $450 \mathrm{~nm}$ was read 300 using a SpectraMAX Plus 384 microplate reader. For IL-1 $\beta$, IL-8, MCP-1, and TNF- $\alpha$ the OD 301 was also read at a reference wavelength of $620 \mathrm{~nm}$, and for IL-6 at a reference wavelength of $302550 \mathrm{~nm}$. For IP-10 and MIP-1 $\alpha$ no reference wavelength was used. Cytokine and chemokine 
concentrations were calculated following the manufacturer's instructions for interpolating

304 unknown concentrations from a standard curve.

\section{Statistical analysis}

307 Unless otherwise noted, data were collected in MS Excel, and statistical analyses were 308 performed using GraphPad Prism software v8 (La Jolla, CA, USA). Statistical details for each 309 experiment are described in the corresponding figure legends. Differences in viral titer, lung 310 injury severity or body weight were analyzed by one-way ANOVA. Differences in the mRNA 311 expression of cytokines were assessed by analyzing replicate data using ordinary one-way 312 ANOVA with the Kruskal-Wallis test or using Student's t-test. Differences in cytokine 313 expression at the protein level were analyzed using unpaired t-test with Welch's correction for 314 normally distributed data, otherwise Mann-Whitney test. Differences in biomarkers were 315 evaluated using Brown-Forsythe ANOVA with the Dunnett T3 correction. P values $<0.05$ were 316 considered statistically significant. 


\section{RESULTS} \\ Zapnometinib inhibits various coronaviruses in vitro.}

320 In our recent publication we demonstrated the antiviral efficacy of zapnometinib against various 321 SARS-CoV-2 strains in cell culture ${ }^{24}$. Here, this in vitro analysis of the antiviral efficacy of 322 zapnometinib against SARS-CoV-2 (BavPat1 and the alpha and beta variants) was extended to 323 other highly pathogenic human coronaviruses (MERS-CoV and SARS-CoV-1) and evaluated using a Virospot reduction assay. A reduction in the viral titer of at least $1 \log _{10}$ in magnitude, correlating to a $90 \%$ reduction, was achieved with $75-100 \mu \mathrm{M}$ zapnometinib compared to the solvent control for all tested viruses (Fig. 1). For SARS-CoV-2 BavPat1 and the beta variant, $100 \mu \mathrm{M}$ zapnometinib achieved a $2-\log _{10}$ reduction (99\%) (Fig. 1 c, d). Furthermore, at $75 \mu \mathrm{M}$, zapnometinib achieved a reduction of more than $80 \%$ for all the viruses tested, while $50 \mu \mathrm{M}$ zapnometinib achieved a reduction of approximately $50 \%$ for SARS-CoV-1 and SARS-CoV-2 BavPat1 (Fig. 1a, c). The $\mathrm{EC}_{50}$ values ranged from $39.0 \mu \mathrm{M}$ (SARS-CoV-1) to $62.6 \mu \mathrm{M}$ (SARS-

CoV-2 beta) for all the viruses tested (Table 1). In summary, in vitro antiviral efficacy of zapnometinib against various coronaviruses could be shown, confirming its broad antiviral therapeutic potential that is, for a host-cell targeting drug, most probably not dependent on upcoming variations due to virus mutations, which is an important drug feature explicitly in the current SARS-CoV-2 pandemic.

\section{Pharmacokinetics of zapnometinib in Syrian hamsters}

338 Next to the in vitro efficacy of a drug, the therapeutic potential and efficacy must be confirmed

339 with in vivo studies. Prior to the in vivo efficacy study, we studied the pharmacokinetics of 340 zapnometinib in Syrian hamsters to better understand the metabolism of zapnometinib in this 341 animal. In addition, the tolerability of zapnometinib was verified by conducting clinical 342 observations. To investigate the dose proportionality, clearance, and safety of zapnometinib in 343 the hamsters used as model animals, a single dose of 15,30 or $60 \mathrm{mg} / \mathrm{kg}$ zapnometinib was 
344 administered p.o. to groups of 6 animals. Blood samples were collected for analysis after 30

345 minutes and $1,2,4,8,12$, and $24 \mathrm{~h}$ from 3 alternating animals per group. The

346 serum concentration time curves for all doses showed a monophasic progression following oral

347 administration. A proportional increase was observed until the maximum observed plasma

348 concentration $\left(\mathrm{C}_{\max }\right)$ was reached after $2 \mathrm{~h}$ for the 15 and $30 \mathrm{mg} / \mathrm{kg}$ doses and after $4 \mathrm{~h}$ for the

$34960 \mathrm{mg} / \mathrm{kg}$ dose (Fig. 2). Moreover, the area under the concentration-time curve (AUC) and

350 the $\mathrm{C}_{\max }$ values increased proportionally with the dose. The apparent terminal half-life was

351 comparable for all dose groups and was in the range of 2-3 h (Table 2). Notably, at the highest

352 dose $(60 \mathrm{mg} / \mathrm{kg})$, the concentration of zapnometinib after $24 \mathrm{~h}$ (prior to the next treatment) was

$3535.32 \mu \mathrm{g} / \mathrm{mL}$ (Table 2; Fig. 2). Based on previous work in our laboratory, $10 \mu \mathrm{g} / \mathrm{mL}$ is required

354 for 50\% MEK inhibition. Thus, higher doses are needed to achieve a serum concentration

355 exceeding $10 \mu \mathrm{g} / \mathrm{mL}$ at $24 \mathrm{~h}$, ensuring effective and constant MEK inhibition. Additionally, all

356 doses were well tolerated, and no adverse events upon zapnometinib treatment were reported.

357 Therefore, based on the present data, a loading dose of $100 \mathrm{mg} / \mathrm{kg}$ followed by a daily dose of

$35875 \mathrm{mg} / \mathrm{kg}$ was used for the efficacy study in the Syrian hamster infection model.

360 Antiviral efficacy of zapnometinib in the Syrian hamster infection model

361 An in vivo study was designed to investigate the efficacy of zapnometinib administered p.o. in

362 SARS-CoV-2-infected hamsters. In this efficacy study, all animals were challenged with $1 \times 10^{3}$

363 TCID 50 SARS-CoV-2 via intranasal administration, which induces both high levels of viral

364 replication in the respiratory tract and significant histopathological changes in the lungs ${ }^{29}$.

365 Control animals were treated with vehicle (5\% DMSO, 15\% Kolliphor in PBS) beginning at 4

366 h p.i., while the experimental animals were treated with the following regimen: one loading

367 dose of $100 \mathrm{mg} / \mathrm{kg}$ zapnometinib (4 or $24 \mathrm{~h}$ p.i.) followed by $75 \mathrm{mg} / \mathrm{kg}$ zapnometinib once per

368 day until the day of sacrifice (4 dpi). During the challenge phase, the animals were weighed, 
clinical observations were monitored upon treatment and infection, and throat swabs were taken

370 daily (Fig. 3a). At 4 dpi, all animals were euthanized for virological and pathological analyses.

371 For the analysis of viral titers, throat swabs were analyzed by titration for the detection of 372 replication competent virus and additionally subjected to TaqMan-based RT-qPCR for the 373 detection of viral RNA to determine the viral copy number. At 2 dpi, the viral load in the throat 374 swabs was comparable among all three treatment groups (Fig. 3b). At 3 dpi, the TCID 50 assay 375 indicated that zapnometinib treatment of both treatment groups resulted in a significant reduction in the amount of replication-competent virus in the throat swabs, with reductions of $1.1 \log _{10}(p=0.0134)$ and $1.3 \log _{10}(p=0.0191)$ noted in the groups treated at 4 h p.i. and 24 h p.i., respectively (Fig. 3b, top row). These results were supported by the RT-qPCR data, which demonstrated viral titer reductions (number of viral copies) of $1.1 \log _{10}(p=0.0089)$ and $3801.2-\log _{10}(p=0.0090)$ in animals treated at $4 \mathrm{~h}$ p.i. and $24 \mathrm{~h}$ p.i., respectively, compared to control animals (Fig. 3b, bottom row). By contrast, at 4 dpi, viral titers were still lower in the throat swabs of drug-treated animals, but the differences were not statistically significant (Fig. $3 b)$.

384 A similar pattern was observed for the infectious viral load in the nasal turbinates at 3854 dpi. Treatment starting at 4 h p.i. significantly reduced the infectious viral titer by $1.3 \log _{10}(p$ $386=0.0335)$. These data were further confirmed by RT-qPCR, which showed a reduction of $3871.1 \log _{10}(p=0.0145)$ when treatment was started at 4 h p.i. (Fig. 3c). However, there was no 388 significant reduction in viral load when treatment was started at $24 \mathrm{~h}$ p.i. .

389 To gain further insight into how treatment affects disease progression beside the influence on 390 viral load, the lung lesions of the infected animals were evaluated by gross pathology at 4 dpi, 391 and the percentage of affected lung area was estimated. The percentage of lung injury in left 392 lobe lung tissue was significantly reduced in both treated groups $(p=0.0282$ for treatment 393 beginning at $4 \mathrm{~h}$ p.i., $p=0.0314$ for treatment beginning at $24 \mathrm{~h}$ p.i.) compared to the vehicle394 treated group, indicating a beneficial effect of zapnometinib treatment (Fig. 3d). Next to the 
gross pathology analysis of the lungs, a more detailed examination was performed.

396 Histopathological evaluation of the lungs revealed that pulmonary changes were mostly mild

397 in all treatment groups, however, inflammation was less severe in the zapnometinib-treated

398 groups than in the vehicle-treated control group (Fig. 3f). Hamsters treated with the vehicle

399 showed alveolitis with many inflammatory cells, including macrophages and neutrophils in the

400 alveolar septa and lumina, hemorrhage and edema in the alveolar lumina and type II

401 pneumocyte hyperplasia. Hamsters treated with zapnometinib exhibited reduced alveolitis with

402 less cellular inflammatory infiltrate containing few macrophages and neutrophils and no

403 hemorrhage, edema, or type II pneumocyte hyperplasia (Fig. 3f). Comparing the lungs of all

404 treatment groups revealed that alveolar edema was less frequent in animals treated beginning

405 at $4 \mathrm{~h}$ p.i. than in control animals and absent in animals treated beginning at $24 \mathrm{~h}$ p.i.

406 Additionally, intra-alveolar hemorrhage, which is suggestive of epithelial damage, was

407 comparable between animals treated beginning at $4 \mathrm{~h}$ p.i. and vehicle-treated animals, whereas

408 it was absent in animals treated beginning at $24 \mathrm{~h}$ p.i. (Fig. $3 \mathrm{~g}$ ). The alveolar epithelium showed

409 variable scoring of hyperplasia among the different treatment groups (type II hyperplasia),

410 which is related to regeneration. Animals treated beginning at $4 \mathrm{~h}$ p.i. were less affected, and

411 no hyperplasia was observed in animals treated beginning at $24 \mathrm{~h}$ p.i. (Fig. $3 \mathrm{~g}$ ).

412 In general, treatment was well tolerated in both groups, as no significant weight differences

413 were observed among all groups; mean body weight loss was higher than 5\% and comparable

414 among all groups (ranging from 8-11\%) (Fig. 3e). In addition, no treatment related adverse

415 effects were observed in the zapnometinib treated groups compared to the vehicle-treated

416 group. Taken together, these results demonstrate that zapnometinib treatment could

417 significantly reduce viral load in the respiratory tract, alleviated lung pathology and was well-

418 tolerated in the Syrian hamster SARS-CoV-2 infection model. 


\section{Levels of drug safety biomarkers}

422 To further evaluate drug safety, serum samples collected pre- ( 0 dpi) and post-infection (4 dpi) and zapnometinib treatment were used for the assessment of candidate protein biomarkers indicating organ injury. Various candidate biomarkers were analyzed to assess drug-induced vascular injury (DIVI) ${ }^{30}$ and drug-induced liver injury (DILI) ${ }^{30,31}$. The levels of high mobility group box 1 (HMGB1; an indicator of necrosis) ${ }^{32,33}$ and glutamate dehydrogenase (GLDH; an indicator of liver damage) ${ }^{34}$ were measured to assess liver injury (DILI), and p-selectin (SELP) was used as an early indicator of endothelial damage to blood vessels (DIVI). There were no prominent fold changes in the levels of the examined biomarkers between before and after infection and treatment of the animals, and the levels were comparable among all groups at each timepoint (Fig. 4). Collectively, these results provide further evidence that the selected treatment regimen (100 mg/kg zapnometinib followed by $75 \mathrm{mg} / \mathrm{kg}$ beginning at $4 \mathrm{or} 24 \mathrm{~h} \mathrm{p.i.)}$ was not toxic to hamsters.

\section{Effect of zapnometinib on the levels of proinflammatory cytokines and chemokines}

Beside demonstrating the antiviral efficacy, we also examined the potential immunomodulatory properties of zapnometinib. In a first approach, we compared the expression of cytokines and chemokines in serum samples from the hamsters of the efficacy study by ELISA, but there were no significant differences among all the groups after infection and treatment at 4 dpi (Supplementary Fig. 1). This was also generally observed in RT-qPCR analysis from lung tissue 441 samples of the same animals at 4 dpi (Supplementary Fig. 2). Thus, the immune dampening 442 effect of zapnometinib could not be shown by comparing serum and lung tissue cytokine and 443 chemokine levels before ( 0 dpi) and after infection and treatment ( 4 dpi) in the Syrian hamster 444 SARS-CoV-2 infection model. For this reason, we used an LPS-induced acute lung injury (ALI) mouse model to mimic the disease severity and to evaluate the anti-inflammatory properties of 446 zapnometinib as well as its capacity to reduce cytokine storm. Using this virus-independent 
model, we could demonstrate the direct effect of zapnometinib on cytokine and chemokine expression. Mice were challenged by i.p. injection of $5 \mathrm{mg} / \mathrm{kg}$ LPS and treated with $25 \mathrm{mg} / \mathrm{kg}$

449 zapnometinib after $1 \mathrm{~h}$ post-stimulation, and alterations in cytokine and chemokine gene 450 expression patterns were evaluated by comparison with untreated control mice in an $\mathrm{RT}^{2}$ 451 profiler PCR array. As shown in a volcano plot, the expression of most of 84 genes that encode 452 for key secreted proteins central to inflammation, like IL-1 $\beta$, IL-6, MCP-1 (CCL2), IL-8 453 (CXCL1), IP-10 (CXCL10) and MIP-1 $\alpha$ (CCL3), was downregulated after zapnometinib 454 treatment compared to control treatment (Fig. 5a). Importantly, the levels of the Ifna2 gene 455 encoding for IFN- $\alpha$, a key regulator of the innate antiviral immune response, were not 456 significantly downregulated $(p=0.118)$. The expression of various important genes known to 457 be elevated in COVID-19, such as $I l 6, C x c l 1$ and $T n f^{35}$ was significantly reduced in the lung 458 tissues of zapnometinib-treated mice (Fig. 5b).

459 In addition to the animal model, we also wanted to show the immunomodulatory properties of 460 zapnometinib in a primary human cell type, namely freshly isolated human PBMCs. PBMCs 461 isolated from two healthy donors were challenged with $1 \mu \mathrm{g} / \mathrm{mL}$ LPS from E. coli O55:B5 462 (prepared in PBS) and treated with $10 \mu \mathrm{g} / \mathrm{mL}$ zapnometinib (prepared in $0.1 \% \mathrm{DMSO}$ ) for $6 \mathrm{~h}$. 463 Cytokine and chemokine expression analysis by ELISA confirmed the anti-inflammatory 464 properties of zapnometinib in human PBMCs. Expression levels of IL-1 $\beta$, IL-6, IL-8, IP-10, 465 MCP-1, MIP-1 $\alpha$, and TNF- $\alpha$ were significantly reduced upon treatment with zapnometinib for $4666 \mathrm{~h}$ compared to the untreated control group (Fig. 5c, Supplementary Fig. 3). Taken together, 467 experiments confirm the anti-inflammatory properties of zapnometinib in vivo and in primary 468 human blood cells, showing significant reduction of cytokines and chemokines that are known 469 to be elevated in COVID-19. 
473 Recent cell culture studies provided first indications that the MEK inhibitor zapnometinib has anti-SARS-CoV-2 activity in vitro and downmodulated expression of candidate cytokines, 475 suggesting that the drug may have a dual benefit in COVID-19. The present work aimed to 476 evaluate whether these in vitro activities would also translate to an impact in an infected 477 organism, indicating whether zapnometinib displays a dual therapeutic effect in vivo. Therefore, 478 we analyzed antiviral efficacy as well as the immunomodulatory impact of zapnometinib in a well-established Syrian hamster SARS-CoV-2 infection model and in other models of hyperinflammatory infectious diseases. Given that in vitro activities of antiviral compounds are not always reflected in vivo, it is highly substantial that a dual effect of zapnometinib could be demonstrated in vivo, as evidenced by reduced levels of both viral RNA and infectious particles in hamster throat swabs and nasal turbinates, as well as an ameliorative effect on lung 484 pathology. Moreover, by using drug-safety biomarkers it could be shown that zapnometinib was not toxic in the hamster model. All the results emphasize the therapeutic potential of zapnometinib against coronavirus infections correlated with hyperinflammatory symptoms. Zapnometinib, a host targeted antiviral (HTA) inhibits the cellular Raf/MEK/ERK signaling 488 pathway that is pivotal for replication of various viruses ${ }^{36}$. As by February 2022, nirmatrelvir, molnupiravir and remdesivir are considered for treatment against COVID-19 ${ }^{16,37-39}$. In the Syrian hamster model of SARS-CoV-2 infection, zapnometinib showed a significant effect on viral load reduction 3 dpi (Fig. 3b) comparable to that of the orally administered nucleoside 492 analog molnupiravir ${ }^{37}$. Furthermore, zapnometinib treatment resulted in a reduction in lung 493 pathology, indicating therapeutic potential. We do not expect zapnometinib, as a host targeted 494 antiviral agent, to exert mutagenic effects on the virus. The mutagenic potential of nucleoside analog antivirals such as remdesivir or molnupiravir in the treatment of viral diseases raises concerns about enhancing viral evolution. This should not occur with host targeted antivirals 497 since they do not interfere with viral RNA replication. Another major advantage of host targeted 498 antivirals is a high barrier towards the emergence of viral resistance since the virus cannot easily 
replace the missing cellular function. Finally, since viral dependence on cellular factors should not rapidly change during pathogen evolution, activity should be preserved also against new and upcoming variants. In line with the claim, it is of importance to stress that zapnometinib was also active against other highly pathogenic human SARS-CoV viruses, such as SARSCoV-1 and MERS-CoV, as well as all tested variants of SARS-CoV-2 (Fig. 1).

504 A major emphasis of the study was to investigate the impact of the immunomodulatory effect 505 of zapnometinib in vivo, as a second beneficial activity beside its antiviral action. We did not observe a significant difference in cytokine and chemokine expression at 4 dpi in the SARSCoV-2 infected Syrian hamster, suggesting that this model may not be suited to represent hyperinflammatory courses of COVID-19. This assumption is in line with a previous study reporting that in the SARS-CoV-2-challenged Syrian hamster model, the levels of the cytokines and chemokines increase only very early after infection in lung tissue and only to a small extent in the serum ${ }^{40}$. Therefore, we selected an in vivo model that on the one hand represents a fulminant hyperinflammatory disease and on the other hand is independent of the dynamics of 513 pathogen infection. The latter model feature is important to discriminate between direct anti514 inflammatory activities or indirect activities primarily due to the reduction of pathogen titers. 515 Acute lung injury (ALI) is an inflammatory condition caused by pathogen infection, sepsis, trauma, ischemia, or reperfusion. The most severe form of ALI is termed acute respiratory

517 distress syndrome (ARDS), which is also a clinical feature in severe COVID-19 ${ }^{41}$. The 518 excessive inflammatory response in the ALI model was induced by bacterial LPS stimulation. 519 Zapnometinib treatment resulted in a massive reduction of hyperinflammatory cytokine 520 responses, indicating the immunomodulatory potency of the drug in vivo.

521 In addition, we analyzed zapnometinib potency in a primary human cell model, namely freshly 522 isolated PBMCs. Cytokine reduction was also observed in PBMCs, demonstrating a similar 523 immunomodulatory effect. These studies emphasize the important anti-inflammatory potential 524 of zapnometinib. 
Dexamethasone, a glucocorticoid that is currently used to treat severe COVID-19, inhibited

526 cytokines production, mainly by interfering with NF- $\kappa \mathrm{B}$ signaling in animal models of LPS-

527 induced ALI and of human PBMCs ${ }^{42,43}$. Dexamethasone also regulates type I interferons 528 (IFNs), the first line of defense against severe viral respiratory infections ${ }^{41,44,45}$. Tocilizumab 529 and baricitinib, two immunomodulatory compounds recommended to treat severe COVID-19, 530 also demonstrated efficacy in dampening various cytokines and chemokines in ALI animal 531 models and in human PBMCs. A clear influence on interference with type I IFN could not be 532 demonstrated. Since both drugs lead to inhibition of NF- $\kappa \mathrm{B}$, an influence on type I IFN can be 533 assumed since NF- $\mathrm{KB}$ is a major regulator of the IFN-beta enhanceosome ${ }^{46}$. In contrast to the 534 NF- $\kappa \mathrm{B}$ cascade, interfering with the Raf/MEK/ERK signaling pathway will not dampen type I 535 IFNs and downstream IFN regulated genes as shown in this report and in our previous work ${ }^{24}$. 536 The studies conducted here show that zapnometinib ${ }_{2}$ in comparison to dexamethasone, tocilizumab and baricitinib, showed a comparable immunomodulatory potential in animal models and in human PBMCs. This illustrates the advantage of MEK-inhibitors dampening the proinflammatory cytokine response that is the cause of severe hyperinflammation, but not the 540 innate antiviral IFN response that is essential as a first line of defense against virus infection. 541 Further clinical studies are needed to confirm these findings.

542 Overall, the study illustrates that zapnometinib might have great therapeutic potential with a 543 novel approach and broad efficacy. This represents a clear benefit over current therapies. The 544 standard therapies target either the virus itself or the hyperinflammatory response. 545 Zapnometinib, by inhibiting the Raf/MEK/ERK signaling pathway, demonstrates a dual effect, 546 namely viral load reduction and dampening of the pro-inflammatory cytokine and chemokine 547 response. This discriminates the drug from most if not all COVID-19 drugs licensed or under 548 development.

549 A phase 2 clinical trial of zapnometinib in hospitalized COVID-19 patients is ongoing 550 (NCT04776044). Its immunomodulatory effect could make zapnometinib superior to other 
antiviral drugs in the treatment of hospitalized patients, in whom the inflammatory response

552 has a strong influence on disease development. Because MEK inhibition leads to modulation 553 rather than full suppression of the immune response ${ }^{47,48}$, zapnometinib might also be effective 554 against post-COVID syndromes associated with SARS-CoV-2 infection. In line with our 555 previous studies ${ }^{24}$, our results presented here underline the value of zapnometinib alone or in 556 combination with other drugs that act at different stages of viral infection and disease 557 progression, which could be the best possible strategy for controlling the disease and might be 558 an important step forward in the current COVID-19 pandemic. On top of that, zapnometinib 559 may still have other implications in infectious diseases. The drug recently received an orphan 560 drug designation for the treatment of hantavirus infections by the FDA and a phase 2 clinical 561 trial against influenza is in preparation. This demonstrates the potential of zapnometinib as a 562 broad acting anti-infective compound against acute viral infections.

\section{FIGURE LEGENDS}

566 Figure 1: Zapnometinib inhibits different coronaviruses in vitro. The dose-dependent 567 inhibitory effect of zapnometinib $(100,75,50$ and $25 \mu \mathrm{M})$ compared to a control (virus-infected 568 cells, not treated) was analyzed using a Virospot reduction assay (four replicates per 569 concentration). The calculated $\mathrm{EC}_{50}$ obtained through this assay are shown in Table 1. Data 570 were obtained after $48 \mathrm{~h}$ of incubation in 3\% serum for all viruses except for SARS-CoV-1 (24 571 h). a SARS-CoV-1 b MERS-CoV c SARS-CoV-2 BavPat1 d SARS-CoV-2 Beta e SARS-CoV5722 Alpha. The data are presented as the mean + SD. The data were analyzed by one-way 573 ANOVA. $* p<0.05, * * * * p<0.0001$

575 Figure 2: Pharmacokinetic analysis of zapnometinib in Syrian hamsters. Three different 576 doses $(15,30$ and $60 \mathrm{mg} / \mathrm{kg})$ of zapnometinib were administered orally to Syrian hamsters $(\mathrm{n}=$ 
6 per group), and serum was collected $0.5,1,2,4,8,12$ and 24 hours later (alternately from 3 animals per group). The zapnometinib concentration in the serum was determined by HPLCMS/MS. The data are presented as mean serum concentration vs._time profiles. The dashed line shows the critical plasma level for MEK inhibition (approx. $10 \mu \mathrm{g} / \mathrm{mL}$ ).

Figure 3: Study of the efficacy of zapnometinib against SARS-CoV-2 in a Syrian hamster infection model. a Study schedule. On day 0 , all animals were infected intranasally with $1 \times 10^{3}$ TCID $_{50}$ SARS-CoV-2. zapnometinib $(100 \mathrm{mg} / \mathrm{kg})$ was given beginning $4 \mathrm{hp}$.i. or $24 \mathrm{~h}$ p.i. Thereafter, the animals in both groups were treated with $75 \mathrm{mg} / \mathrm{kg}$ zapnometinib once daily until sacrifice. The control group received vehicle (5\% DMSO, 15\% Kolliphor, $80 \%$ PBS) starting 4 h p.i. Body weight was measured, and throat swabs were taken daily. At 4 dpi, all animals were sacrificed. Nasal turbinate tissues were subsequently homogenized and subjected to virus titration. Blood was collected for further analysis, the lungs were inspected, and the percentage of affected lung tissue was estimated from the dorsal view. The left lung lobe was collected for histopathological examination. The study schedule was created on biorender.com.

b Infectious viral titers in throat swabs collected at 2, 3 and 4 dpi expressed as $\log _{10}$ TCID $_{50}$ of control (vehicle-treated) and zapnometinib-treated SARS-CoV-2-infected hamsters (upper row) or expressed as genome copies per g determined by RT-qPCR (bottom row). c Infectious viral loads in nasal turbinates collected from control (vehicle-treated) and zapnometinib-treated SARS-CoV-2-infected hamsters at 4 dpi expressed as $\log _{10} \mathrm{TCID}_{50}$ per g nasal turbinate tissue analyzed via the $\mathrm{TCID}_{50}$ assay (left) or as copies per g determined by PCR (right). The dashed lines represent the lower limit of detection $\left(0.8 \log _{10} \mathrm{TCID} \mathrm{D}_{50} / \mathrm{mL}\right.$ for titration and $1.9 \log _{10}$ $\mathrm{CP} / \mathrm{mL}$ for RT-qPCR). d Percentage of lung area with pathological signs in control (vehicletreated) and zapnometinib-treated SARS-CoV-2-infected hamsters at 4 dpi. e Weight loss at 4 dpi as a percentage of the body weight measured at the time of infection. Individual data 
603 (dots) and median values (horizontal lines) are presented. The bars represent the min. and max.

604 values. f Histopathologic changes at 4 dpi in the lungs of hamsters infected with SARS-CoV-2 605 and treated with zapnometinib or treated with the vehicle only. Hamsters treated with the 606 vehicle showed alveolitis with hemorrhage (arrowheads) and edema (*) in the alveolar lumina 607 and type II pneumocyte hyperplasia (arrows) (A and C). Hamsters treated with zapnometinib 608 exhibited reduced alveolitis with less cellular inflammatory infiltrate and no hemorrhage, 609 edema, or type II pneumocyte hyperplasia (B and D). Representative images for each group. 610 Hematoxylin and eosin staining; 40x magnification (A and C) and 200x magnification (B and

611 D). $\mathrm{g}$ Lung slides were examined and scored for the presence of alveolar hemorrhage, edema 612 and type II pneumocyte hyperplasia $(0=$ no, $1=$ yes $)$. Mean and individual values are presented. 613 All data are from a single experiment. The number of animals per group was as follows: $\mathrm{n}_{\text {(Vehicle) }}$ $614=6 ; \mathrm{n}_{\text {(Treatment }+4 \text { h p.i.) }}=6 ; \mathrm{n}_{\text {(Treatment }+24 \text { h p.i.) }}=5$. The data were analyzed by one-way ANOVA. * $615 p<0.05$.

617 Figure 4: Candidate safety biomarker analysis. The levels of candidate biomarkers in serum 618 samples from hamsters collected before infection with SARS-CoV-2 (0 dpi, prior to infection 619 and zapnometinib treatment) and at 4 dpi and after zapnometinib treatment were analyzed by 620 IA-LC-MS/MS. High mobility group box 1 protein (HMGB1, an indicator of necrosis), 621 glutamate dehydrogenase (GLDH, an indicator of liver damage), and p-selectin (SELP, an 622 indicator of endothelial damage) levels in the zapnometinib-treated and vehicle-treated groups 623 are shown. The treatment conditions described below the graphs refer to the treatment that the 624 animals received after infection. Individual data and median values are presented. The number 625 of animals per group was as follows: $\mathrm{n}_{\text {(Vehicle })}=6 ; \mathrm{n}_{\text {(Treatment }+4 \text { h p.i.) }}=6 ; \mathrm{n}_{\text {(Treatment }+24 \text { h p.i. })}=5$. One 626 data point from the vehicle-treated group is not depicted in the graph showing GLDH levels 627 (4 dpi) because it was outside of the axis limit $(252 \mathrm{ng} / \mathrm{mL})$. The data were analyzed by one628 way ANOVA. P values $<0.05$ were considered statistically significant. 
630 Figure 5: The levels of cytokines and chemokines associated with COVID-19 are reduced

631 after treatment with zapnometinib a, b mRNA expression of different cytokines and 632 chemokines in acute lung injury (ALI) model mice after LPS (5 mg/kg) stimulation and 633 zapnometinib treatment $(25 \mathrm{mg} / \mathrm{kg}) 1 \mathrm{~h}$ post stimulation, while the mice in the control group 634 were treated with vehicle (1\% DMSO in PBS). The mRNA levels were determined at $6 \mathrm{~h}$ post 635 treatment by an $\mathrm{RT}^{2}$ profiler PCR array. a The volcano plot shows genes that showed significant expression changes. Representative genes mainly associated with COVID-19 were highlighted. The $\log _{2}$ of the fold change in gene expression is plotted on the $\mathrm{x}$-axis, and statistical significance is plotted on the y-axis. The two dashed vertical lines indicate the fold change threshold, while the horizontal line indicates the $\mathrm{p}$ value threshold. Regardless of statistical significance, red and blue indicate upregulation and downregulation, respectively, while black indicates no change in gene expression. Genes represented by data points in the far upper left (downregulated) and far upper right (upregulated) sections met the fold change and p value thresholds, while the genes represented by the data points between the two vertical lines showed 644 no change in gene expression. b Representative expression. Mean values + SD are shown. The 645 data were analyzed using the $\Delta \Delta \mathrm{Ct}$ method and normalized to the mean expression levels of 5 646 housekeeping genes. The $\mathrm{P}$ values for the difference in replicate (-DeltaCt) expression data for 647 each gene between the control and treatment groups were calculated by Student's t-test. ** $p<$ $6480.01, * * * * p<0.0001$. c Reduction in cytokine expression after zapnometinib treatment in 649 LPS-stimulated human PBMCs from one healthy donor. Cells were simultaneously stimulated 650 with $1 \mu \mathrm{g} / \mathrm{mL}$ LPS and treated with $10 \mu \mathrm{g} / \mathrm{mL}$ zapnometinib for $6 \mathrm{~h}$. The expression of cytokines 651 in the supernatants was analyzed by ELISA. The data are presented as the mean \pm SD of

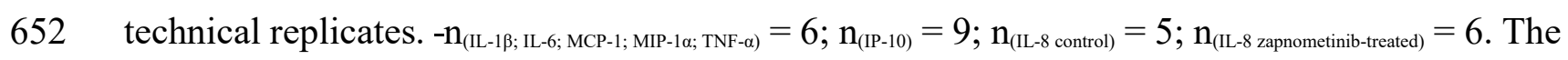
653 differences in the levels of all cytokines of interest between the control and zapnometinib- 
654 treated groups were analyzed with unpaired t-test with Welch's correction. $* * p<0.01$;

$655 p<0.0001$.

656

657 T-values and degrees of freedom: IL-1b: $\mathrm{t}=25.08, \mathrm{df}=3.509$. IL-6: $\mathrm{t}=18.61, \mathrm{df}=2.036$. IL-8:

$658 \mathrm{t}=8.733, \mathrm{df}=2.001$. IP-10: $\mathrm{t}=15.95, \mathrm{df}=2.049$. MCP-1: $\mathrm{t}=56.76, \mathrm{df}=2.305$, MIP-1a: $\mathrm{t}=10.04$,

$659 \mathrm{df}=2.012$. TNF-a: $\mathrm{t}=7.261, \mathrm{df}=3.771$ (ALI mouse model).

660 IL-1b: $\mathrm{t}=12.11, \mathrm{df}=9.089$. IL-6: $\mathrm{t}=15.20, \mathrm{df}=9.996$. IL-8: $\mathrm{t}=5.024, \mathrm{df}=4.783$. IP-10: $\mathrm{t}=13.26$,

$661 \mathrm{df}=11.01$. MCP-1: $\mathrm{t}=14.22, \mathrm{df}=5.028$, MIP-1a: $\mathrm{t}=14.75, \mathrm{df}=9.941$. TNF-a: $\mathrm{t}=4.544, \mathrm{df}=6.154$

662 (PBMCs).

663

664

665

ACKNOWLEDGEMENTS

666 The authors thank Annika Schönsiegel for coordinating the shipment of the compound and

667 Eugenia Steinhilber for help with the manuscript. This work was supported by DFG grants 668 SFB1009B13 and CRU342 P6.

669

670

671 AUTHOR CONTRIBUTIONS

672 Conceptualization: Y.F., SL and O.P.; methodology: G.A., H.H., L.W., L.dW.; formal analysis:

673 Y.F., O.P., L.dW., H.H., L.W., A.S., S.S.; investigation: H.H., L.W., L.dW.; histology: JvdB., 674 M.D. resources: O.P.; writing - original draft: Y.F. and O.P.; writing - review \& editing: 675 H.H., L.W., O.Poe., V.A., A.S., L.dW., JvdB., Y.F., SL and O.P.; visualization: H.H., L.W., 676 Y.F., O.P.; supervision: Y.F. and O.P.; project administration: Y.F. and O.P.; funding 677 acquisition: O.P.

678

679 
681 O. Planz has an ownership interest in Atriva Therapeutics $\mathrm{GmbH}$ and is a consultant for Atriva 682 Therapeutics GmbH. Y. Füll and L. Waidele are employees of Atriva Therapeutics GmbH.

\section{REFERENCES}

6861 Khan, M. et al. COVID-19: A Global Challenge with Old History, Epidemiology and Progress So Far. Molecules 26, doi:10.3390/molecules26010039 (2020).

6882 O'Driscoll, M. et al. Age-specific mortality and immunity patterns of SARS-CoV-2.

689 Nature 590, 140-145, doi:10.1038/s41586-020-2918-0 (2021).

6903 Alwani, M. et al. Sex-based differences in severity and mortality in COVID-19. Rev $691 \quad$ Med Virol 31, e2223, doi:10.1002/rmv.2223 (2021).

6924 Burkert, F. R., Lanser, L., Bellmann-Weiler, R. \& Weiss, G. Coronavirus Disease 693 2019: Clinics, Treatment, and Prevention. Front Microbiol 12, 761887, 694 doi:10.3389/fmicb.2021.761887 (2021).

6955 Wang, L. L., Yang, J. W. \& Xu, J. F. Coronavirus (SARS-CoV-2) causes lung 696 inflammation and injury. Clin Microbiol Infect, doi:10.1016/j.cmi.2021.11.022 (2021).

6976 Leisman, D. E. et al. Cytokine elevation in severe and critical COVID-19: a rapid 698 systematic review, meta-analysis, and comparison with other inflammatory 699 syndromes. Lancet Respir Med 8, 1233-1244, doi:10.1016/S2213-2600(20)30404-5 $700 \quad$ (2020).

7017 Fan, S. et al. Neurological Manifestations in Critically Ill Patients With COVID-19: A 702 Retrospective Study. Front Neurol 11, 806, doi:10.3389/fneur.2020.00806 (2020). 7038 Aggarwal, G. et al. Association of Cardiovascular Disease With Coronavirus Disease 2019 (COVID-19) Severity: A Meta-Analysis. Curr Probl Cardiol 45, 100617, 705 doi:10.1016/j.cpcardiol.2020.100617 (2020). 
7069 Shi, W., Lv, J. \& Lin, L. Coagulopathy in COVID-19: Focus on vascular thrombotic 707 events. J Mol Cell Cardiol 146, 32-40, doi:10.1016/j.yjmcc.2020.07.003 (2020).

70810 Gustine, J. N. \& Jones, D. Immunopathology of Hyperinflammation in COVID-19. 709 Am J Pathol 191, 4-17, doi:10.1016/j.ajpath.2020.08.009 (2021).

71011 Costela-Ruiz, V. J., Illescas-Montes, R., Puerta-Puerta, J. M., Ruiz, C. \& Melguizo711 Rodriguez, L. SARS-CoV-2 infection: The role of cytokines in COVID-19 disease. 712 Cytokine Growth Factor Rev 54, 62-75, doi:10.1016/j.cytogfr.2020.06.001 (2020). 71312 Group, R. C. et al. Dexamethasone in Hospitalized Patients with Covid-19. N Engl J $714 \quad$ Med 384, 693-704, doi:10.1056/NEJMoa2021436 (2021).

71513 Goletti, D. \& Cantini, F. Baricitinib Therapy in Covid-19 Pneumonia - An Unmet $716 \quad$ Need Fulfilled. N Engl J Med 384, 867-869, doi:10.1056/NEJMe2034982 (2021).

71714 Stone, J. H. et al. Efficacy of Tocilizumab in Patients Hospitalized with Covid-19. N 718 Engl J Med 383, 2333-2344, doi:10.1056/NEJMoa2028836 (2020).

71915 Yoon, J. J. et al. Orally Efficacious Broad-Spectrum Ribonucleoside Analog Inhibitor 720 of Influenza and Respiratory Syncytial Viruses. Antimicrob Agents Chemother 62, 721 doi:10.1128/AAC.00766-18 (2018).

72216 Owen, D. R. et al. An oral SARS-CoV-2 M(pro) inhibitor clinical candidate for the 723 treatment of COVID-19. Science, eab14784, doi:10.1126/science.abl4784 (2021).

72417 Chang, F. et al. Signal transduction mediated by the Ras/Raf/MEK/ERK pathway 725 from cytokine receptors to transcription factors: potential targeting for therapeutic 726 intervention. Leukemia 17, 1263-1293, doi:10.1038/sj.leu.2402945 (2003).

72718 Lieske, N. V., Tonby, K., Kvale, D., Dyrhol-Riise, A. M. \& Tasken, K. Targeting 728 Tuberculosis and HIV Infection-Specific Regulatory T Cells with MEK/ERK 729 Signaling Pathway Inhibitors. PLoS One 10, e0141903, 730 doi:10.1371/journal.pone.0141903 (2015). 
73119 Nakayama, T. \& Yamashita, M. The TCR-mediated signaling pathways that control

732 the direction of helper T cell differentiation. Semin Immunol 22, 303-309,

733 doi:10.1016/j.smim.2010.04.010 (2010).

73420 Verma, V. et al. MEK inhibition reprograms CD8(+) T lymphocytes into memory

735 stem cells with potent antitumor effects. Nat Immunol 22, 53-66, doi:10.1038/s41590-

$736 \quad$ 020-00818-9(2021).

$73721 \quad$ Pleschka, S. et al. Influenza virus propagation is impaired by inhibition of the

738 Raf/MEK/ERK signalling cascade. Nat Cell Biol 3, 301-305, doi:10.1038/35060098

$739 \quad(2001)$

74022 Laure, M. et al. Antiviral efficacy against influenza virus and pharmacokinetic

741 analysis of a novel MEK-inhibitor, ATR-002, in cell culture and in the mouse model.

$742 \quad$ Antiviral Res 178, 104806, doi:10.1016/j.antiviral.2020.104806 (2020).

74323 Hamza, H., Shehata, M. M., Mostafa, A., Pleschka, S. \& Planz, O. Improved in vitro

744 Efficacy of Baloxavir Marboxil Against Influenza A Virus Infection by Combination

745 Treatment With the MEK Inhibitor ATR-002. Front Microbiol 12, 611958,

746 doi:10.3389/fmicb.2021.611958 (2021).

74724 Schreiber, A. et al. The MEK1/2-inhibitor ATR-002 efficiently blocks SARS-CoV-2

748 propagation and alleviates pro-inflammatory cytokine/chemokine responses. Cell Mol

749 Life Sci 79, 65, doi:10.1007/s00018-021-04085-1 (2022).

75025 Zielinska, E. et al. Development of an improved microneutralization assay for

751 respiratory syncytial virus by automated plaque counting using imaging analysis. Virol

$752 \quad J 2,84$, doi:10.1186/1743-422X-2-84 (2005).

75326 Corman, V. M. et al. Detection of 2019 novel coronavirus (2019-nCoV) by real-time

754 RT-PCR. Euro Surveill 25, doi:10.2807/1560-7917.ES.2020.25.3.2000045 (2020). 
75527 Steinhilber, A. E. et al. Mass Spectrometry-Based Immunoassay for the Quantification 756 of Banned Ruminant Processed Animal Proteins in Vegetal Feeds. Anal Chem 90, 757 4135-4143, doi:10.1021/acs.analchem.8b00120 (2018).

75828 Anselm, V. et al. Matrix and Sampling Effects on Quantification of Protein 759 Biomarkers of Drug-Induced Liver Injury. J Proteome Res 20, 4985-4994, 760 doi:10.1021/acs.jproteome.1c00478 (2021).

76129 Munoz-Fontela, C. et al. Animal models for COVID-19. Nature 586, 509-515, 762 doi:10.1038/s41586-020-2787-6 (2020).

76330 Bendjama, K. et al. Translation strategy for the qualification of drug-induced vascular 764 injury biomarkers. Toxicol Pathol 42, 658-671, doi:10.1177/0192623314527644 $765 \quad$ (2014).

76631 Church, R. J. et al. Candidate biomarkers for the diagnosis and prognosis of drug767 induced liver injury: An international collaborative effort. Hepatology 69, 760-773, 768 doi:10.1002/hep.29802 (2019).

76932 Scaffidi, P., Misteli, T. \& Bianchi, M. E. Release of chromatin protein HMGB1 by 770 necrotic cells triggers inflammation. Nature 418, 191-195, doi:10.1038/nature00858 (2002).

33 Zemskova, M. et al. Necrosis-Released HMGB1 (High Mobility Group Box 1) in the Progressive Pulmonary Arterial Hypertension Associated With Male Sex. Hypertension 76, 1787-1799, doi:10.1161/HYPERTENSIONAHA.120.16118 (2020).

77534 Schomaker, S. et al. Serum glutamate dehydrogenase activity enables early detection of liver injury in subjects with underlying muscle impairments. PLoS One 15, e0229753, doi:10.1371/journal.pone.0229753 (2020).

77835 Del Valle, D. M. et al. An inflammatory cytokine signature predicts COVID-19 severity and survival. Nat Med 26, 1636-1643, doi:10.1038/s41591-020-1051-9 (2020). 
78136 Pleschka, S. RNA viruses and the mitogenic Raf/MEK/ERK signal transduction $782 \quad$ cascade. Biol Chem 389, 1273-1282, doi:10.1515/BC.2008.145 (2008).

78337 Rosenke, K. et al. Orally delivered MK-4482 inhibits SARS-CoV-2 replication in the 784 Syrian hamster model. Nat Commun 12, 2295, doi:10.1038/s41467-021-22580-8 $785 \quad(2021)$

78638 Abdelnabi, R. et al. Comparing infectivity and virulence of emerging SARS-CoV-2 787 variants in Syrian hamsters. Ebiomedicine 68, 103403, 788 doi:10.1016/j.ebiom.2021.103403 (2021).

78939 Ye, Z. W. et al. Beneficial effect of combinational methylprednisolone and remdesivir 790 in hamster model of SARS-CoV-2 infection. Emerg Microbes Infect 10, 291-304, $791 \quad$ doi:10.1080/22221751.2021.1885998(2021).

$79240 \quad$ Nouailles, G. et al. Temporal omics analysis in Syrian hamsters unravel cellular 793 effector responses to moderate COVID-19. Nat Commun 12, 4869, 794 doi:10.1038/s41467-021-25030-7 (2021).

79541 Sinha, S. et al. Dexamethasone modulates immature neutrophils and interferon 796 programming in severe COVID-19. Nat Med 28, 201-211, doi:10.1038/s41591-021$797 \quad 01576-3(2022)$.

79842 Mokra, D., Mikolka, P., Kosutova, P. \& Mokry, J. Corticosteroids in Acute Lung 799 Injury: The Dilemma Continues. Int J Mol Sci 20, doi:10.3390/ijms20194765 (2019).

80043 Held, H. D., Boettcher, S., Hamann, L. \& Uhlig, S. Ventilation-induced chemokine 801 and cytokine release is associated with activation of nuclear factor-kappaB and is $802 \quad$ blocked by steroids. Am J Respir Crit Care Med 163, 711-716, 803 doi:10.1164/ajrccm.163.3.2003001 (2001).

80444 Gessani, S., McCandless, S. \& Baglioni, C. The glucocorticoid dexamethasone 805 inhibits synthesis of interferon by decreasing the level of its mRNA. J Biol Chem 263, $806 \quad$ 7454-7457 (1988). 
80745 McCoy, C. E., Carpenter, S., Palsson-McDermott, E. M., Gearing, L. J. \& O'Neill, L.

808 A. Glucocorticoids inhibit IRF3 phosphorylation in response to Toll-like receptor-3

809 and -4 by targeting TBK1 activation. J Biol Chem 283, 14277-14285,

810 doi:10.1074/jbc.M709731200 (2008).

81146 Hiscott, J. Convergence of the NF-kappaB and IRF pathways in the regulation of the 812 innate antiviral response. Cytokine Growth Factor Rev 18, 483-490, 813 doi:10.1016/j.cytogfr.2007.06.002 (2007).

81447 Wang, Z. Q., Wu, D. C., Huang, F. P. \& Yang, G. Y. Inhibition of MEK/ERK 1/2 815 pathway reduces pro-inflammatory cytokine interleukin-1 expression in focal cerebral 816 ischemia. Brain Res 996, 55-66, doi:10.1016/j.brainres.2003.09.074 (2004).

81748 Pinto, R. et al. Inhibition of influenza virus-induced NF-kappaB and Raf/MEK/ERK 818 activation can reduce both virus titers and cytokine expression simultaneously in vitro 819 and in vivo. Antiviral Res 92, 45-56, doi:10.1016/j.antiviral.2011.05.009 (2011).

820 
Figure 1: in vitro efficacy

a

b

SARS-CoV-1

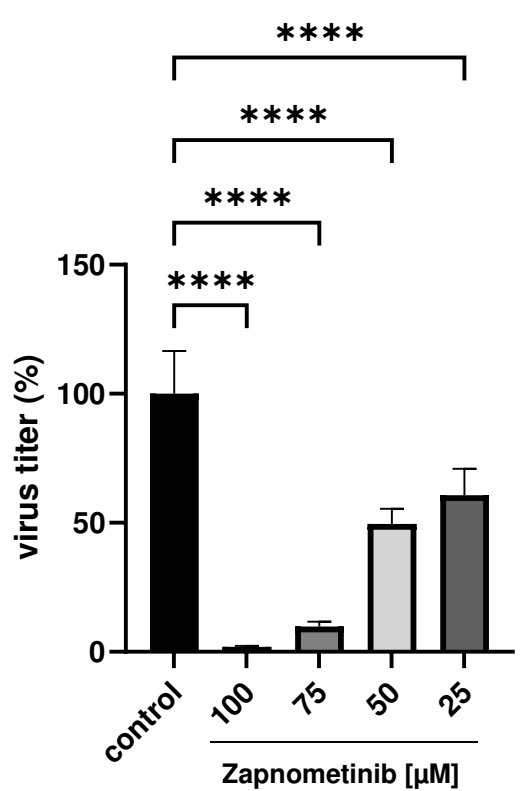

C

MERS-CoV

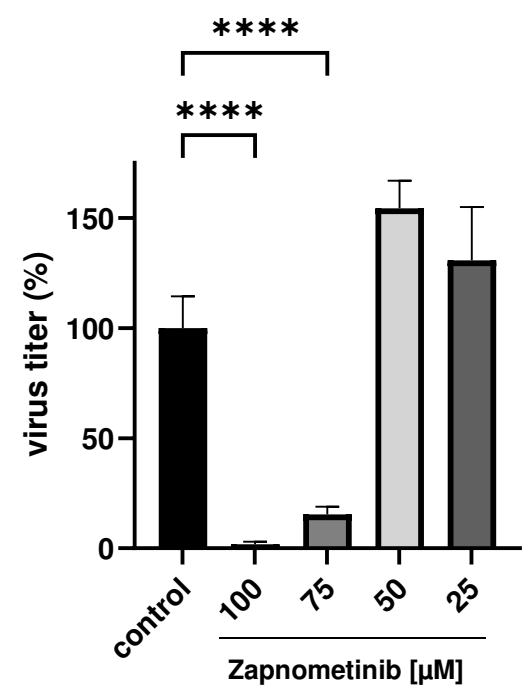

d

SARS-CoV-2 BavPat1

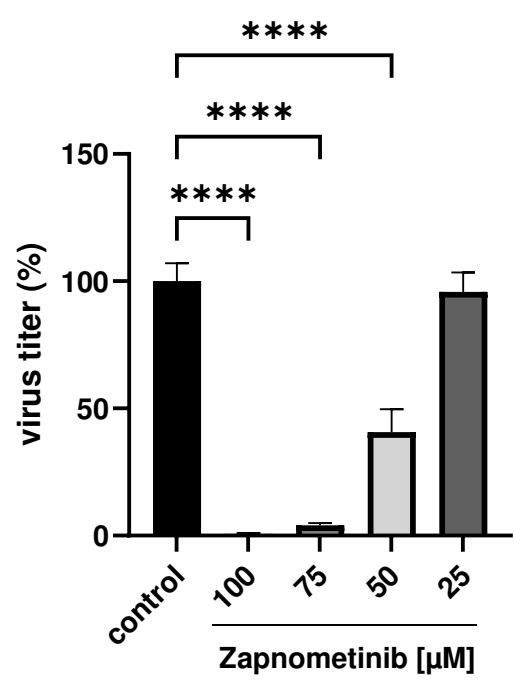

e

SARS-CoV-2 Alpha
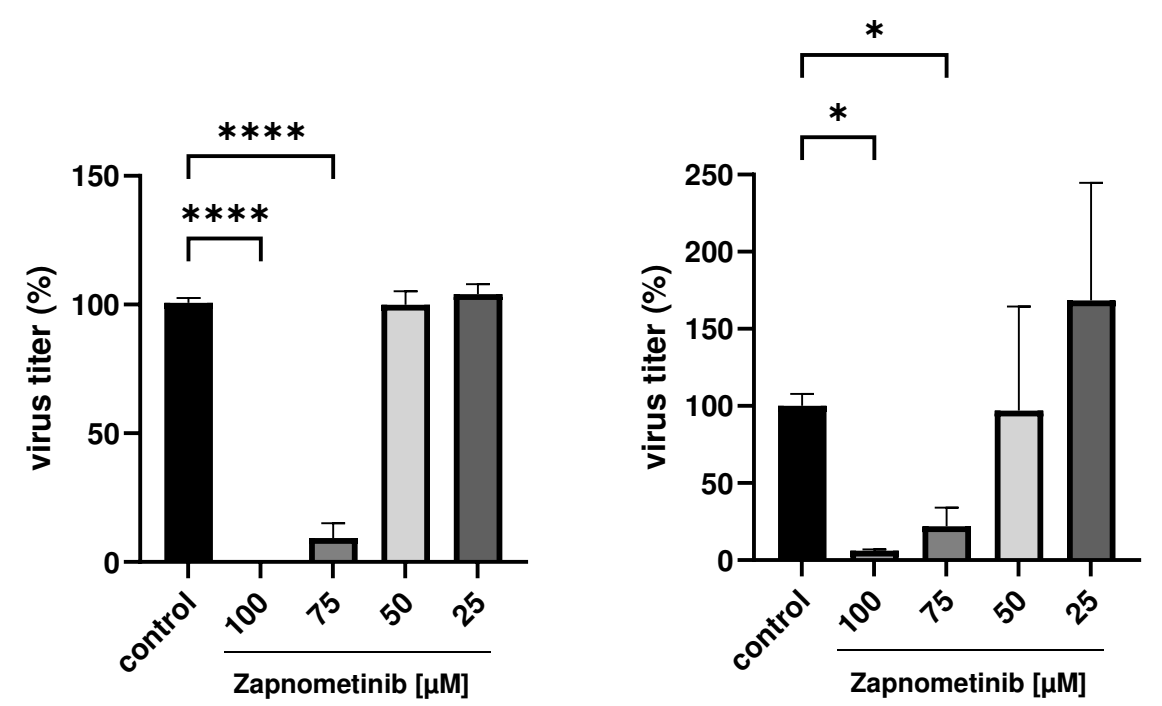


\section{Figure 2: Pharmacokinetics}

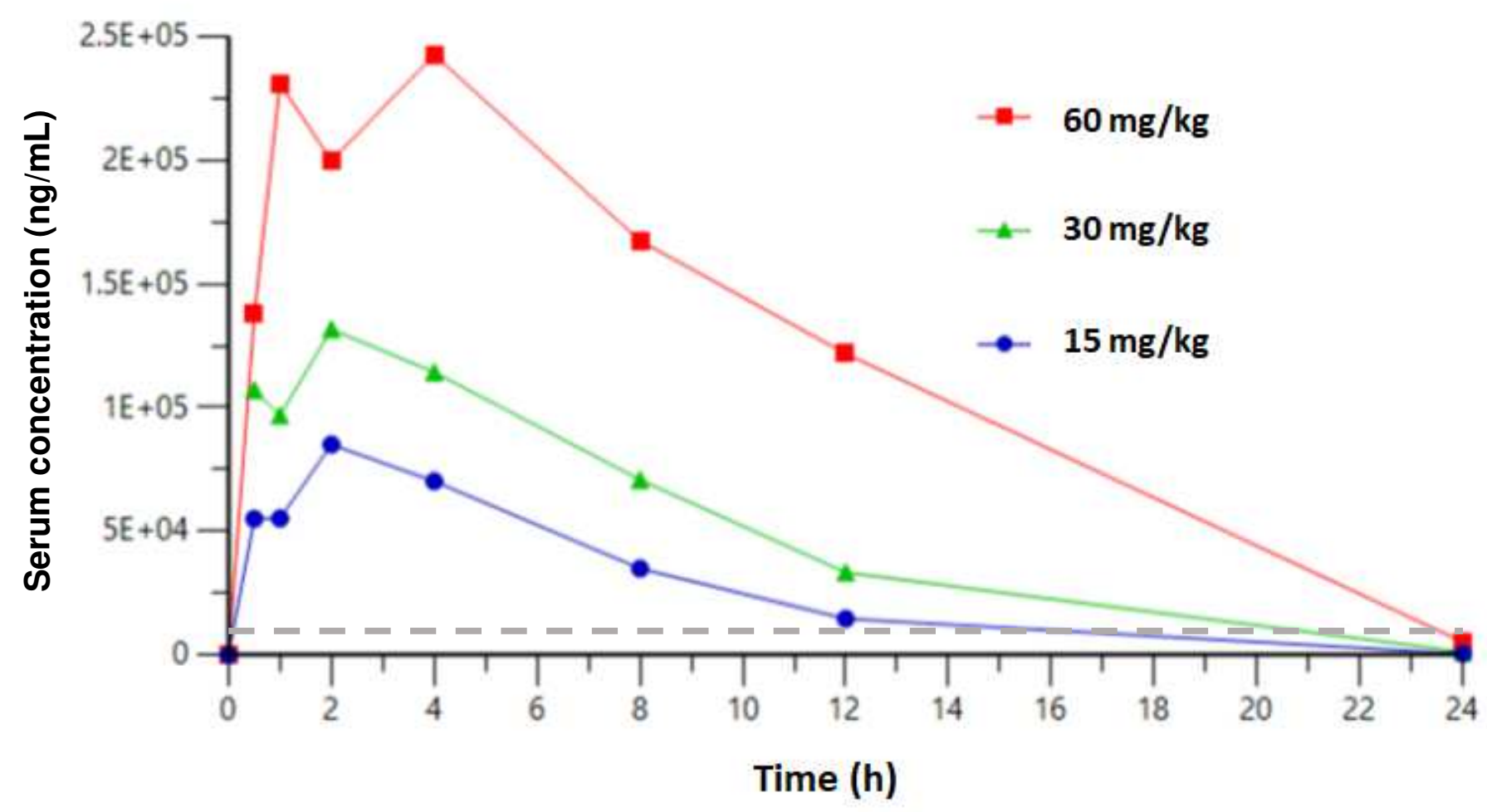




\section{Figure 3: Efficacy study}

a

Treatment start $4 \mathrm{~h}$ p.i. $\quad$ (also control group that received vehicle)

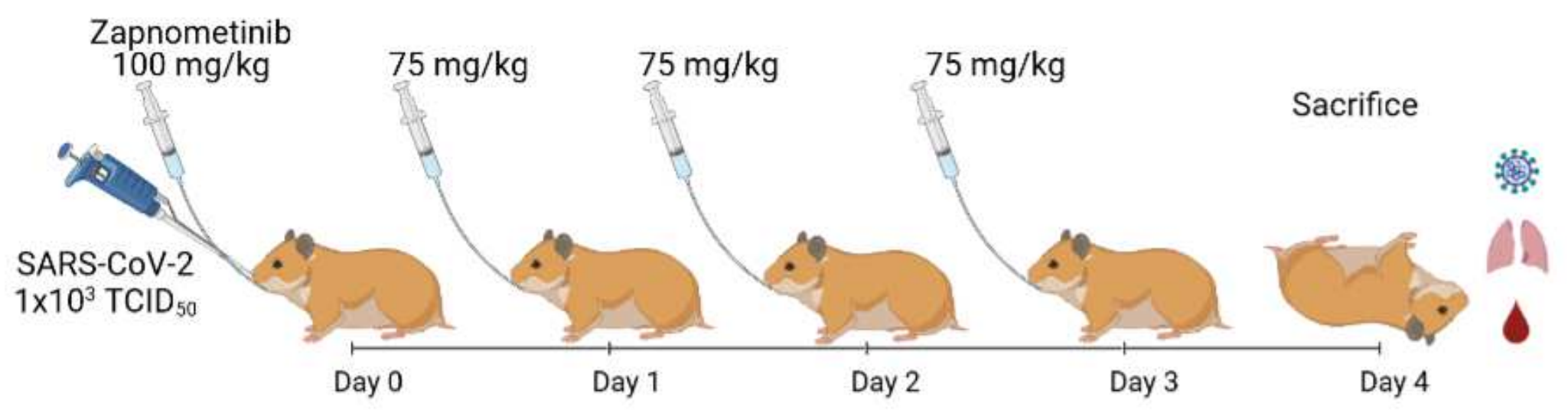

Treatment start $24 \mathrm{~h}$ p.i.

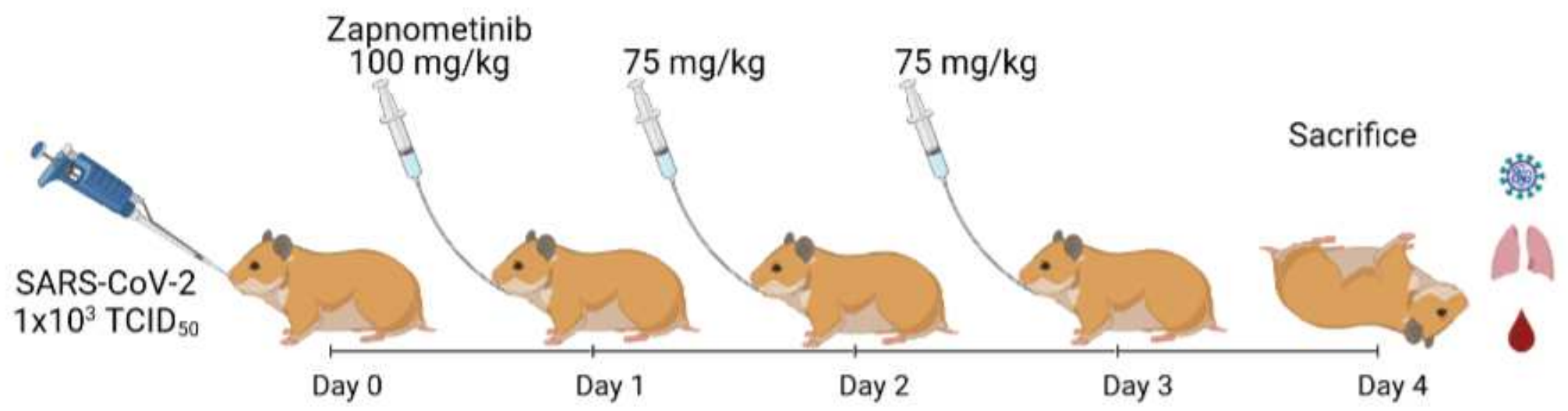

All groups:

Daily: Body weight; Throat swabs Final: Viral load in respiratory tract; Blood sampling; Lung pathology 
Throat swab viral titer $\left(\operatorname{TCID}_{50}\right)$

$2 \mathrm{dpi}$

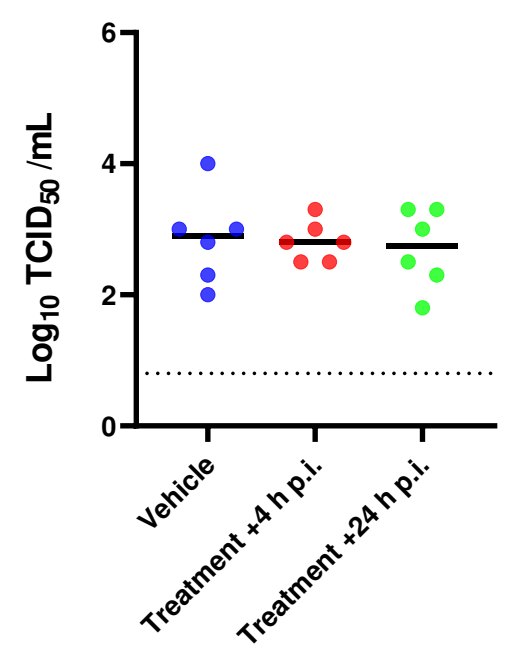

Throat swab viral titer (RT-qPCR)

2 dpi

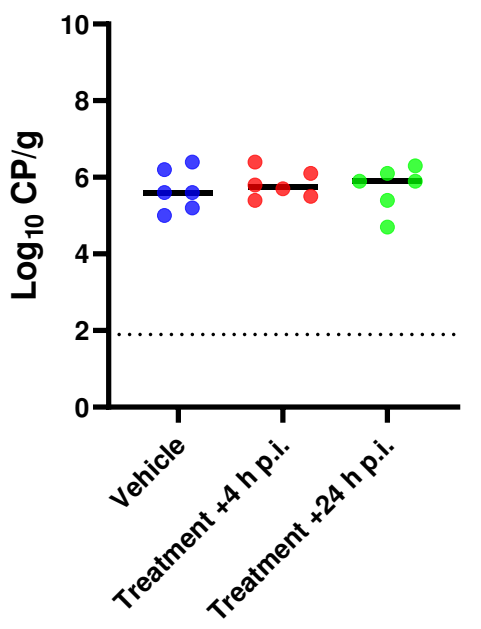

3 dpi
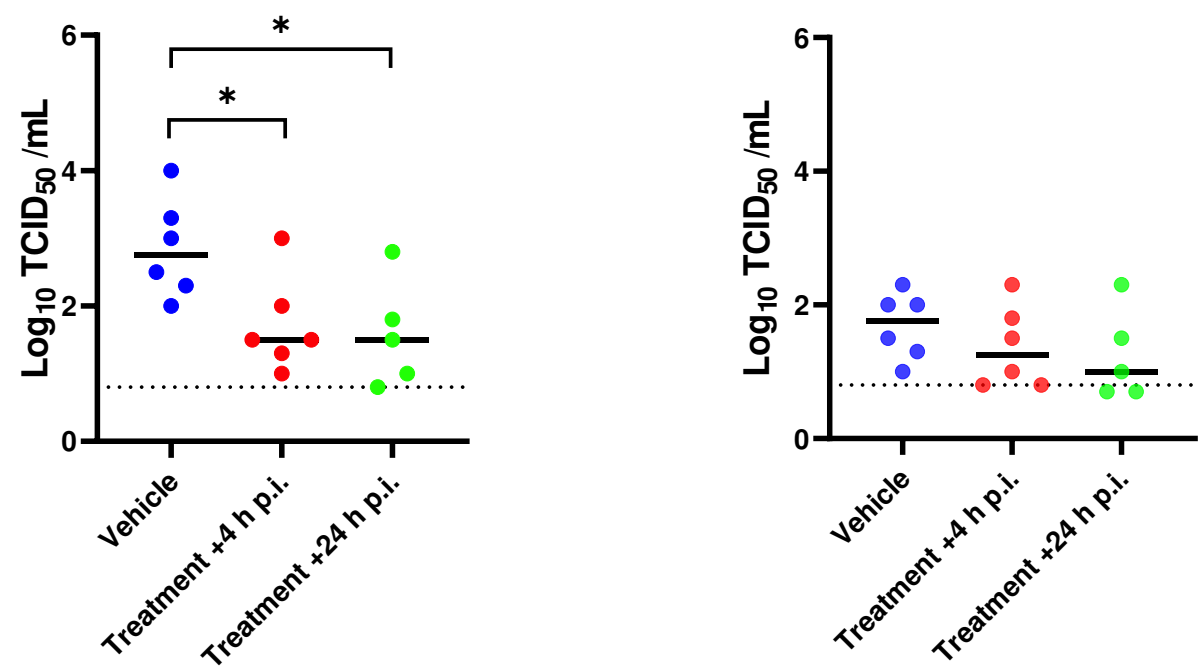

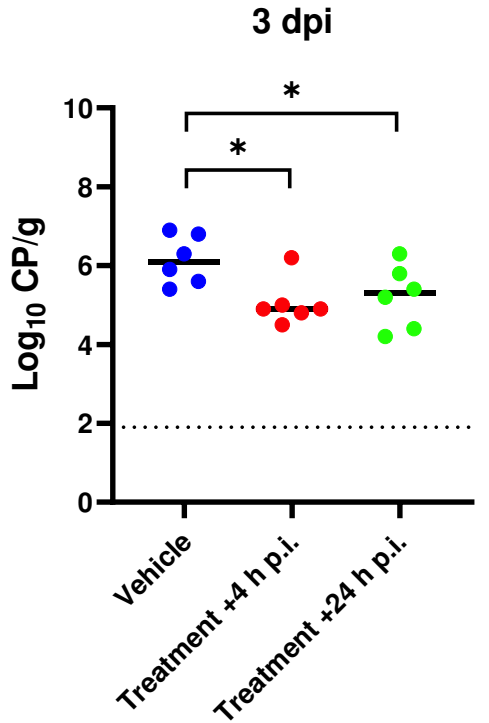

4 dpi

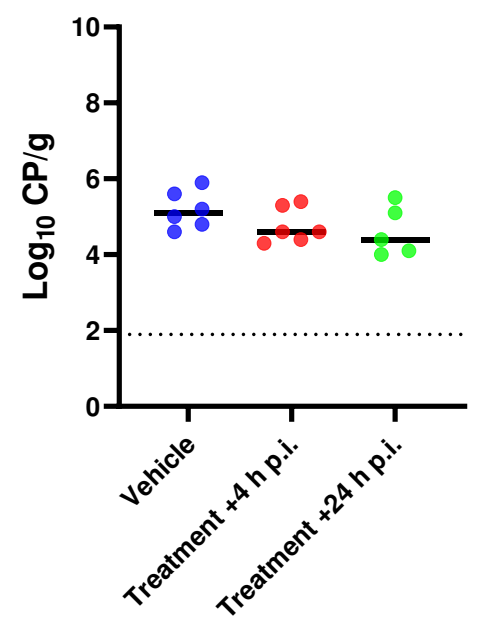




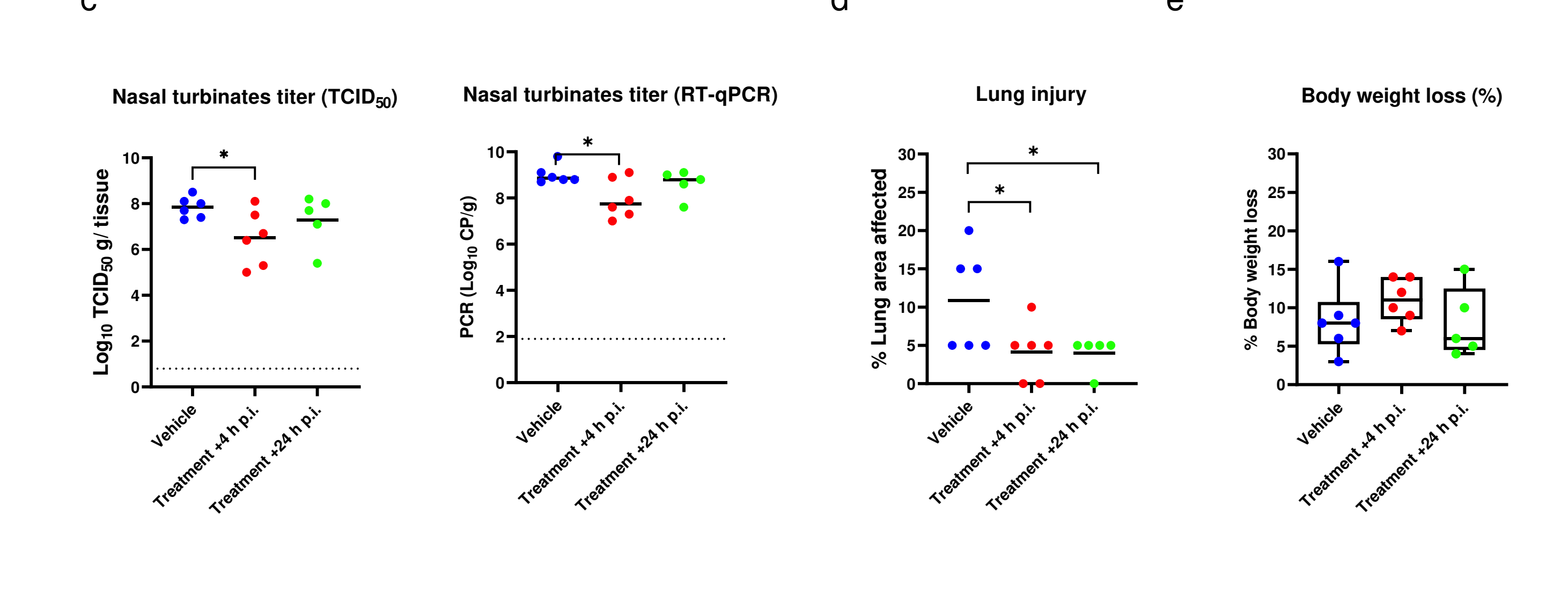




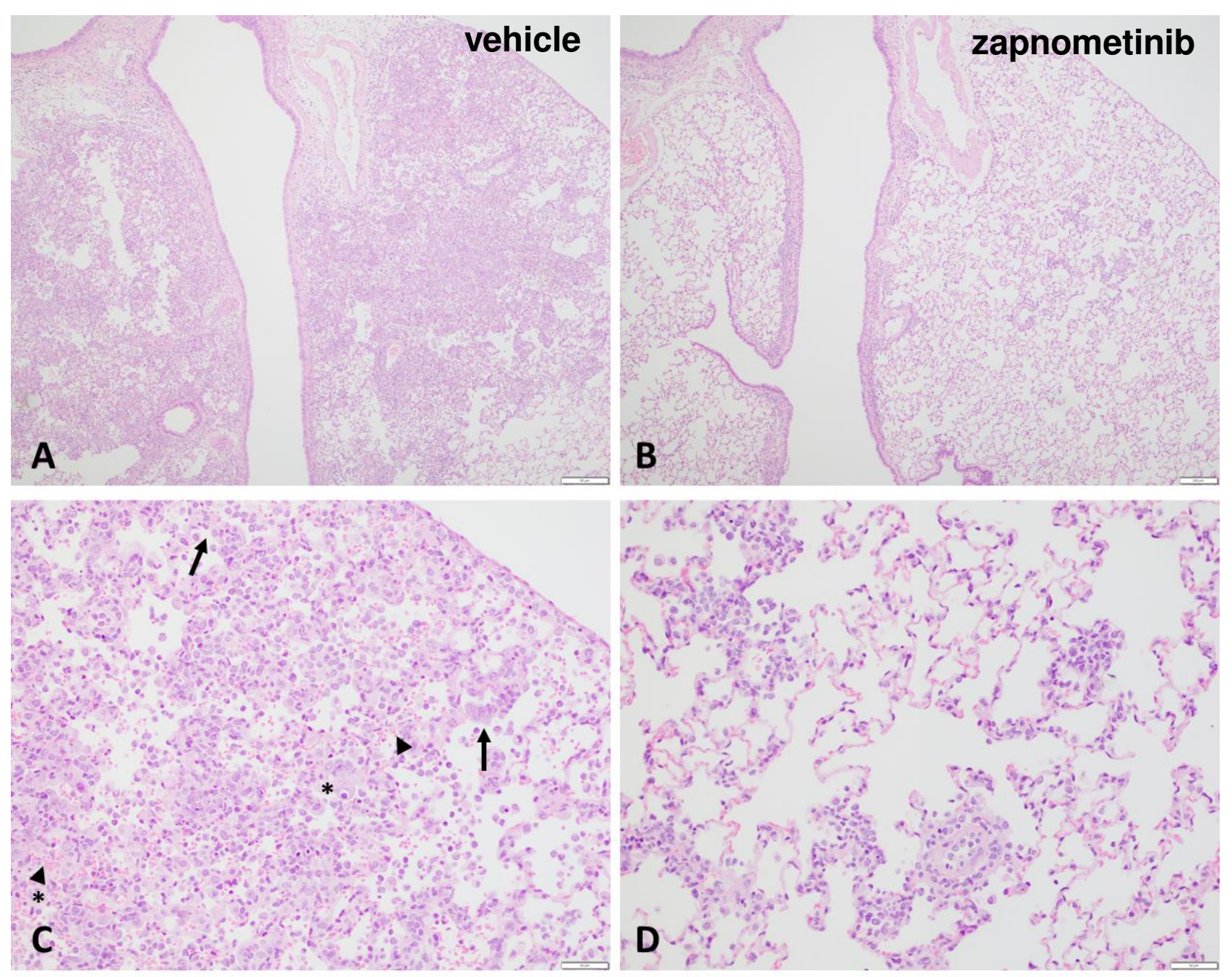



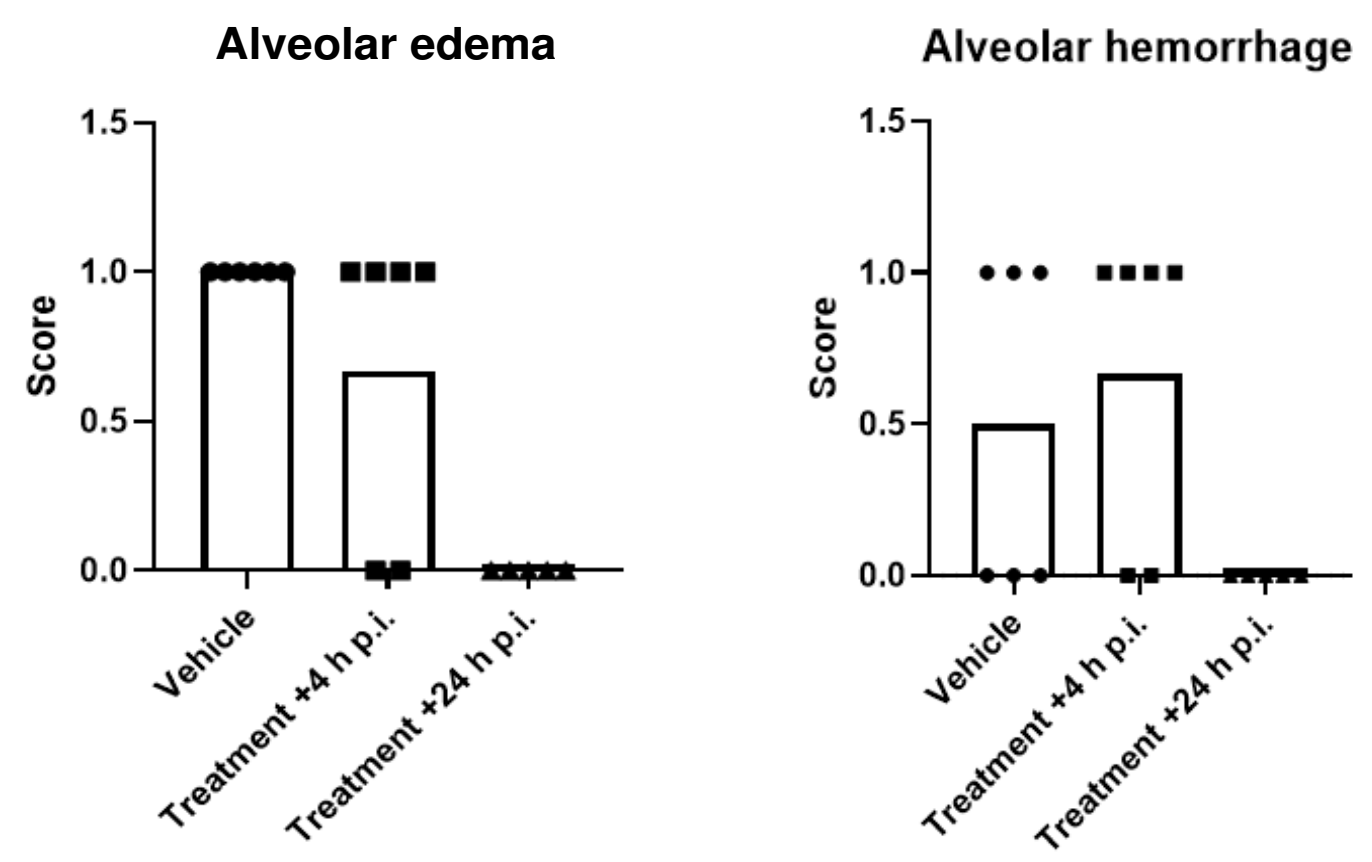

Type II pneumocyte hyperplasia

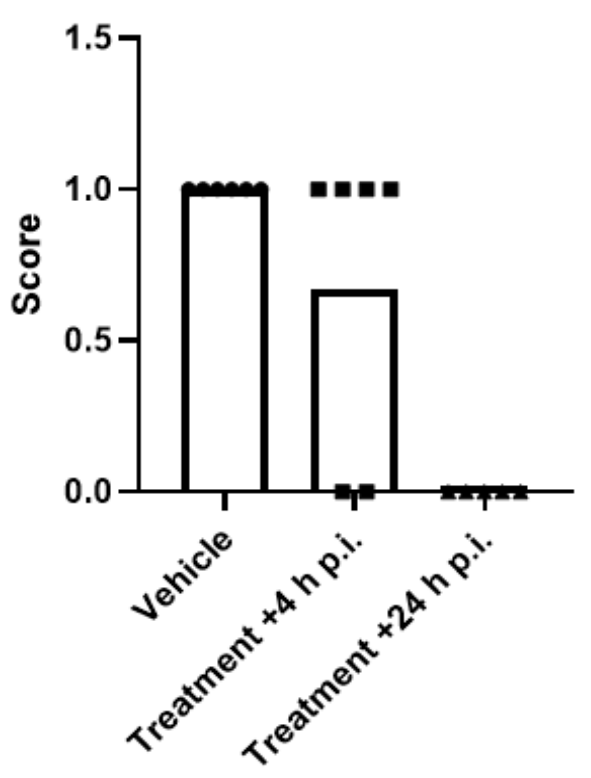




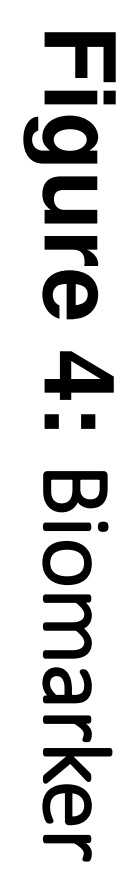

응

응

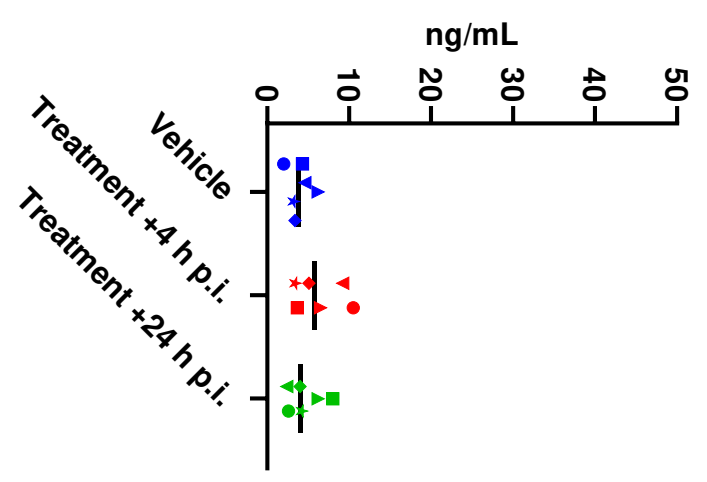

졸

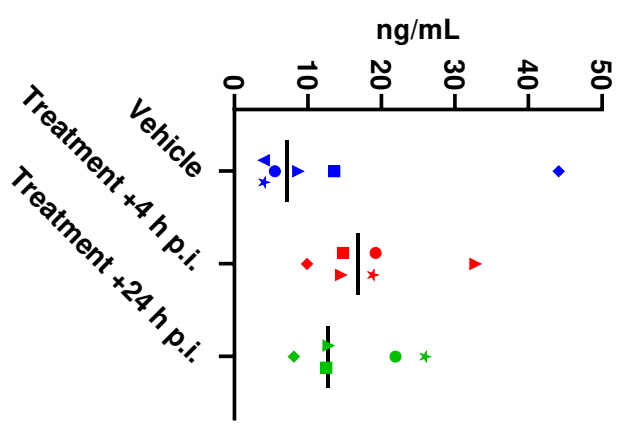

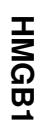

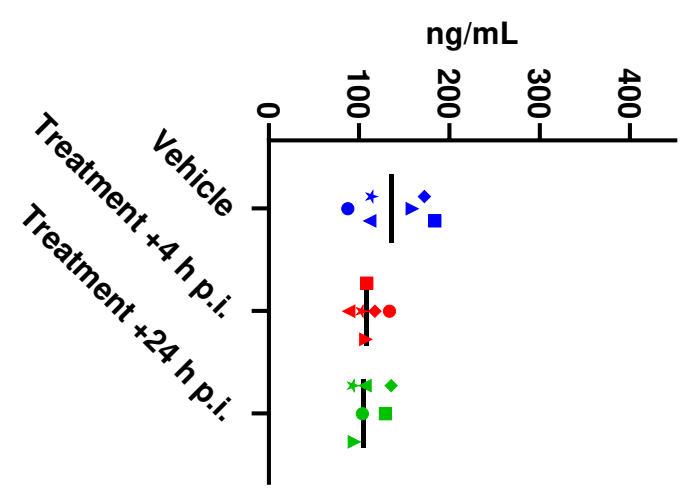

管

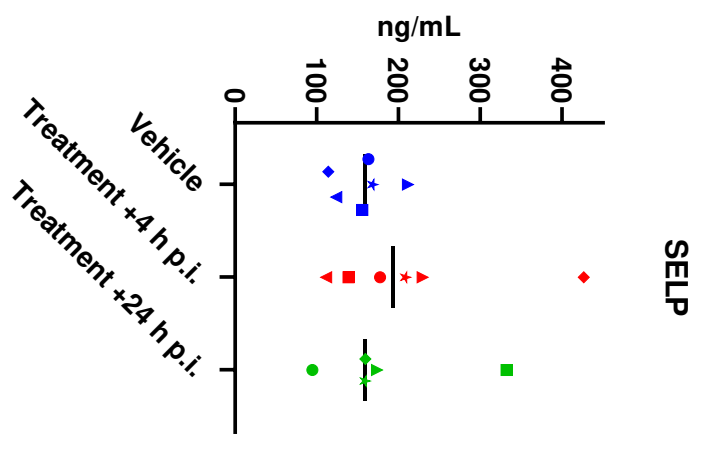

$\mathrm{ng} / \mathrm{mL}$

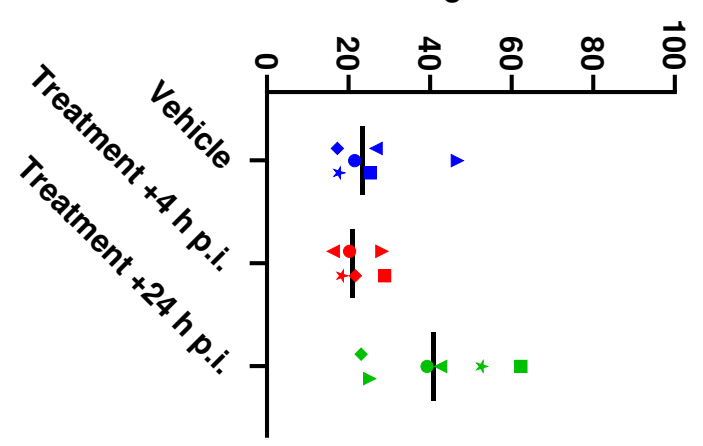

옴

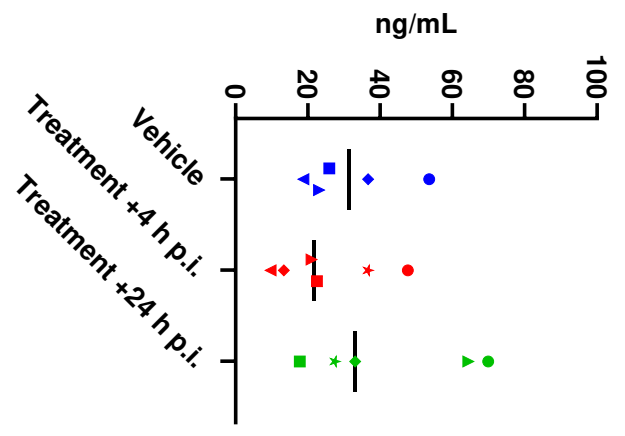

옴 
Figure 5: ALI Mouse Model

Zapnometinib vs. Control

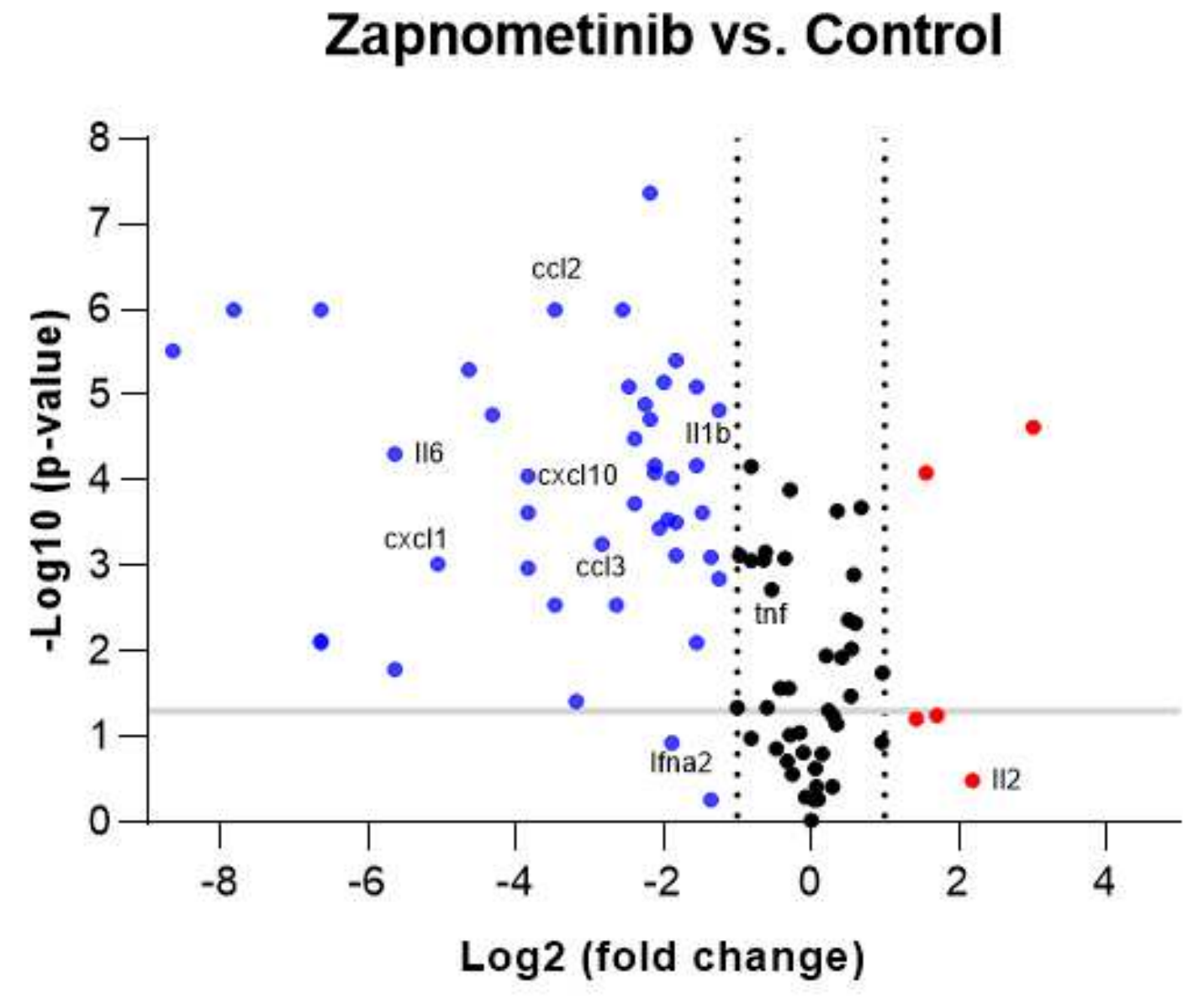

, 
Figure 5: ALI mouse

b

MCP-1 (CCL2)

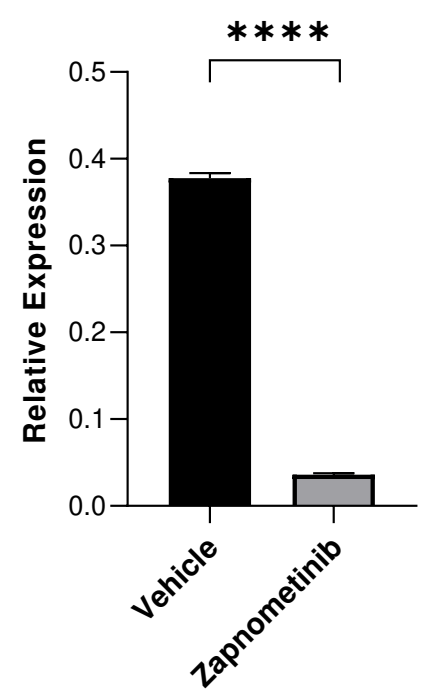

IL-6

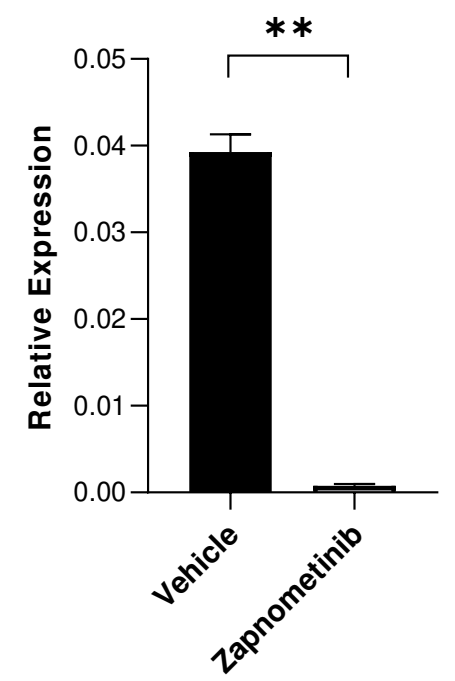

TNF- $\alpha$

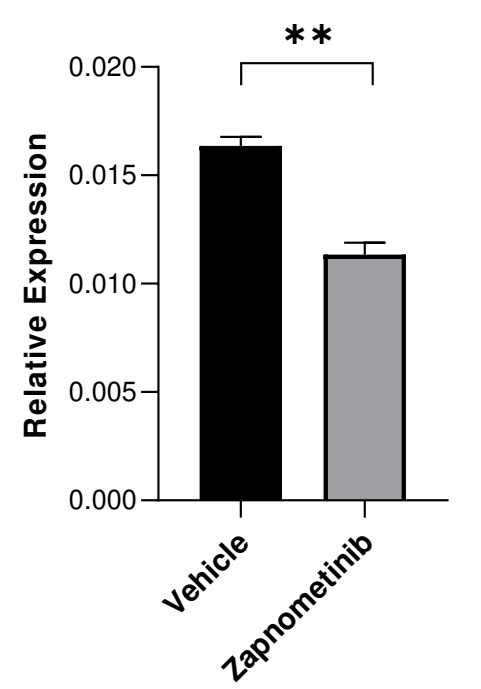

IL-1 $\beta$

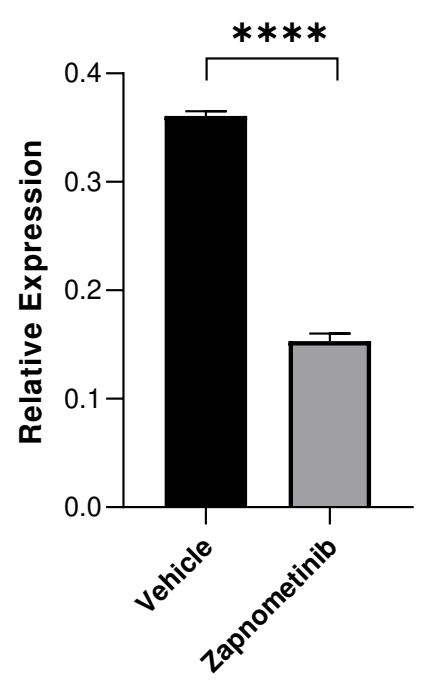

IP-10 (CXCL10)

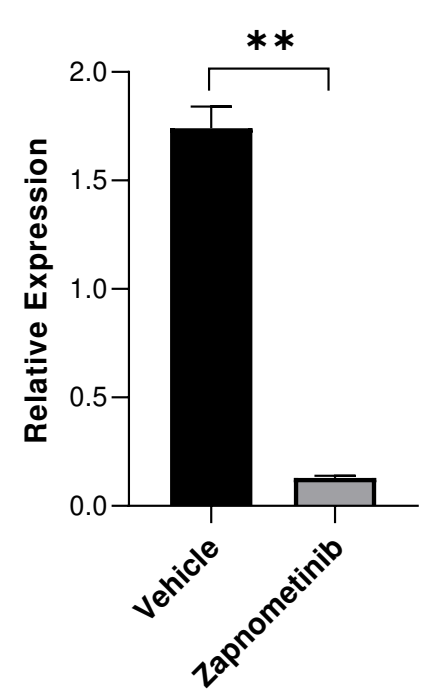

IL-8 (CXCL1)

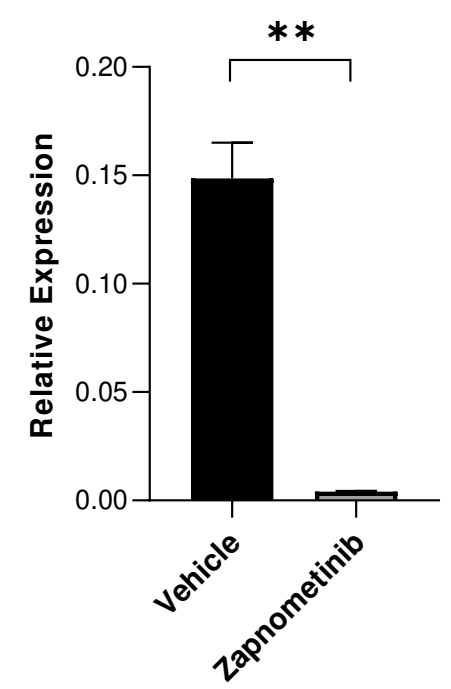

MIP1-a (CCL3)

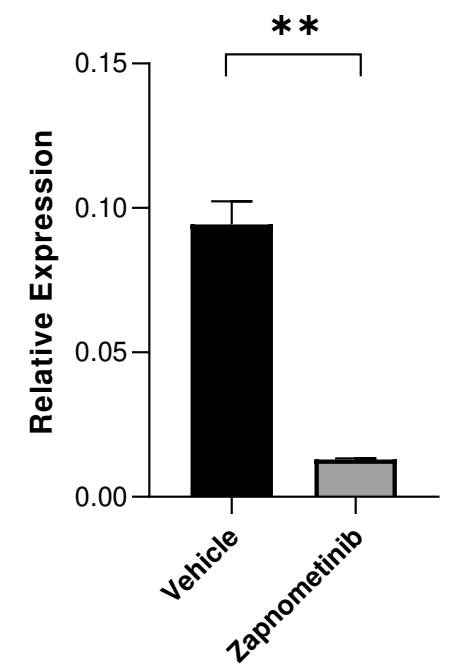


Figure 5: Cytokines PBMCs
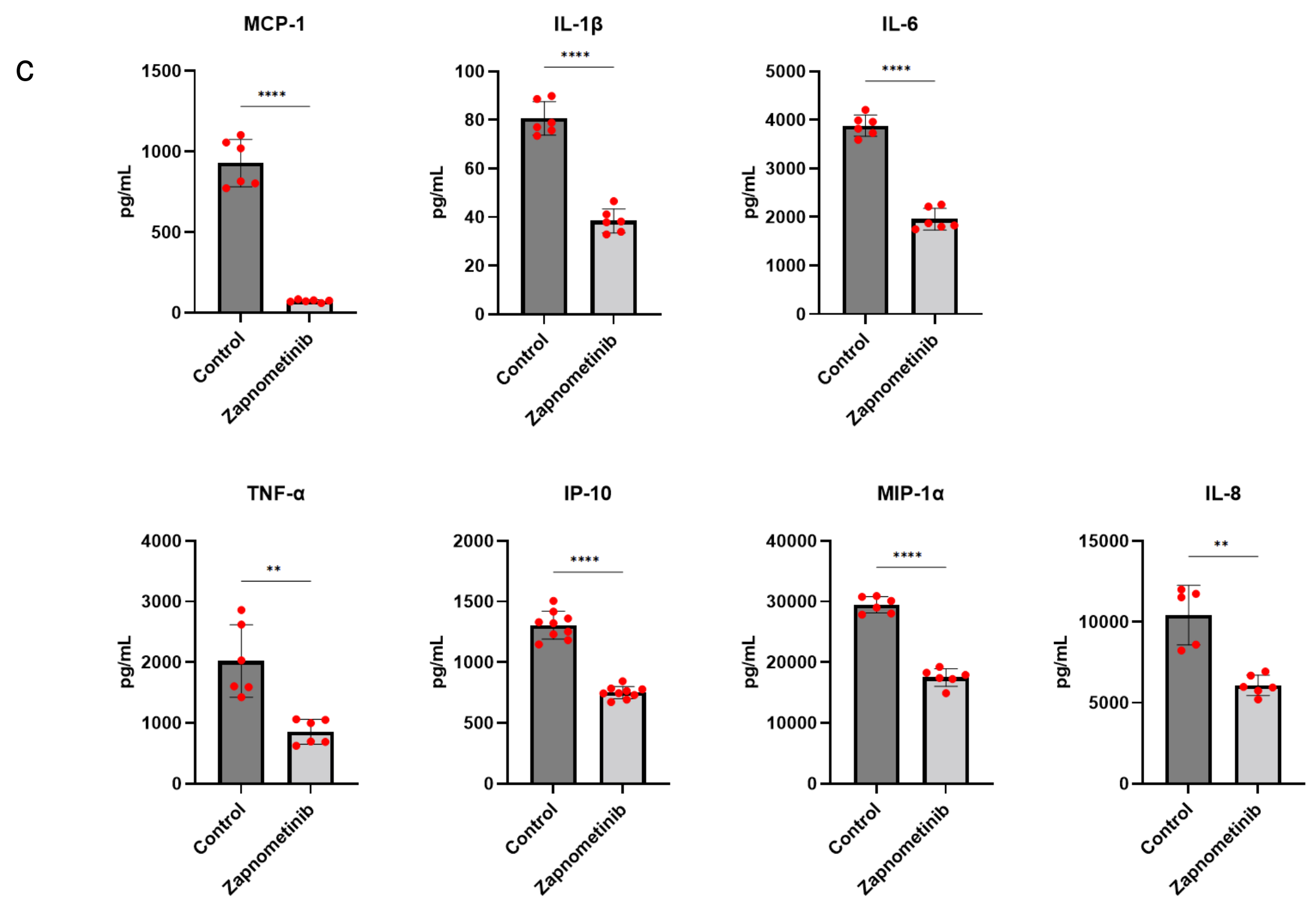


\section{Supplementary Files}

This is a list of supplementary files associated with this preprint. Click to download.

- Fuelletalsupplementary.pdf

- FuelletalsupplementaryTable3GenestableRT2Array.xlsx 